Pacific Journal of Mathematics

TOPOLOGICAL PROPERTIES OF BANACH SPACES

ald arthur Edgar and Robert Francis Wheeler 


\title{
TOPOLOGICAL PROPERTIES OF BANACH SPACES
}

\author{
G. A. Edgar AND R. F. WheELer
}

Let $X$ be a Banach space, $B_{X}$ its closed unit ball. We study several topological properties of $B_{X}$ with its weak topology. In particular, we consider spaces $X$ such that $\left(B_{X}\right.$, weak) is a Polish topological space. If $X$ has RNP and $X^{*}$ is separable, then $B_{x}$ is Polish; if $B_{X}$ is Polish, then $X$ is somewhat reflexive. We also consider spaces $X$ such that every closed subset of ( $B_{X}$, weak) is a Baire space. This is equivalent to property (PC), studied by Bourgain and Rosenthal.

1. Introduction. Let $X$ be a Banach space, and $B_{X}$ the closed unit ball. It is well-known that $B_{X}$ is metrizable in the relative weak topology of $X$ precisely when the dual space $X^{*}$ is norm separable. Moreover, ( $B_{X}$, weak) is compact metrizable precisely when $X$ is separable and reflexive. Here we address an intermediate question: When is ( $B_{X}$, weak) completely metrizable?

TheOREM A. Let $X$ be a separable Banach space. Then the following are equivalent: (1) ( $B_{X}$, weak) is completely metrizable; (2) ( $B_{X}$, weak) is a Polish space; (3) $X$ has property (PC) and is an Asplund space; (4) ( $B_{X}$, weak) is metrizable, and every closed subset of it is a Baire space.

Property (PC) states that for every weakly closed bounded subset $A$ of $X$, the identity map ( $A$, weak $) \rightarrow(A$, norm) has at least one point of continuity. (The letters "PC" stand for "point of continuity".) Property (PC) is a consequence of the Radon-Nikodym property.

Examples of spaces satisfying Theorem A include: spaces with separable second dual (such as separable quasi-reflexive spaces), the predual of the James Tree space [30], [38] (but not JT itself), and the James-Lindenstrauss spaces JL $(S)$ modelled on separable Banach spaces $S$ [29], [36]. On the other hand, if $X$ contains an isomorphic copy of $c_{0}$ or $l^{1}$, then $\left(B_{X}\right.$, weak) is not Polish. An example of Bourgain and Delbaen [5] satisfies Theorem A, but has dual space isomorphic to $l^{1}$.

There is a natural non-metrizable version of the Polish property. A completely regular Hausdorff space $T$ is said to be $\check{C}$ ech complete iff it admits a complete sequence $\left(\mathscr{U}_{n}\right)$ of open covers. Here completeness is defined as follows: if $\mathscr{F}$ is a family of closed subsets of $T$ such that $\mathscr{F}$ has 
the finite intersection property, and $\mathscr{F}$ is $\mathscr{U}_{n}$-small for all $n$ (that is, there exists $F_{n} \in \mathscr{F}$ and $U_{n} \in \mathscr{U}_{n}$ with $F_{n} \subseteq U_{n}$ ), then $\cap \mathscr{F} \neq \varnothing$. Completely metrizable spaces are Cech complete (take $\mathscr{U}_{n}$ to be the family of open sets with diameter less than $1 / n$ ). Locally compact Hausdorff spaces are also Čech complete (take each $\mathscr{U}_{n}$ to be the family of relatively compact open sets). On the other hand, the usual proofs of the Baire Category Theorem can be easily adapted to show that every Čech complete space is a Baire space (the countable intersection of dense open sets is dense). A space $T$ is Čech complete if and only if $T$ is a $G_{\delta}$ set in some (every) compactification of $T$. A metrizable space is Cech complete if and only if it is completely metrizable. Čech completeness is preserved by closed subspaces, $G_{\delta}$ subspaces, and countable products. (See [18, p. 142], [19], [1].)

If $X$ is an infinite-dimensional Banach space, then ( $X$, weak) is not a Baire space, since each multiple of $B_{X}$ is closed and nowhere dense. We shall be interested in spaces $X$ such that $\left(B_{X}\right.$, weak) is a Čech complete topological space. For this to occur, it is necessary and sufficient that $B_{X}$ be a $G_{\delta}$ in $\left(B_{X^{* *}}\right.$, weak $\left.{ }^{*}\right)$, or that $B_{X^{* *}} \backslash B_{X}$ be weak* $\sigma$-compact, or that $X$ be a $G_{\delta}$ in $\left(X^{* *}\right.$, weak*). (Thus the property is preserved by linear homeomorphisms.) We will say that $X$ has Polish ball, if ( $B_{X}$, weak) is a complete separable metric space under some metric. Note that if $\left(x_{n}^{*}\right)$ is a norm dense sequence in $B_{X^{*}}$, then

$$
d(x, y)=\sum_{n=1}^{\infty} 2^{-n}\left|x_{n}^{*}(x)-x_{n}^{*}(y)\right|
$$

is a metric for ( $B_{X}$, weak). However, this metric is not complete unless $X$ is reflexive; in fact, the completion of $\left(B_{X}, d\right)$ is $B_{X^{* *}}$ in a natural way.

If $X$ is separable, then $X$ has Čech complete ball if and only if $X$ has Polish ball (see 3.1 and 3.2, below). In this case, $X^{*}$ is also separable.

THEOREM B. Let $X$ be a Banach space. The following are equivalent: (1) $X$ has Čech complete ball; (2) $X$ is isomorphic to a direct sum $R \oplus S$, where $R$ is reflexive and $S$ has Polish ball.

Theorems A and B yield structural information about spaces with Čech complete ball. Every space with Čech complete ball is weakly compactly generated (WCG), somewhat reflexive, and has property (PC). If $X$ has Čech complete ball, then $X^{*}$ is WCG and has the Radon-Nikodým property (RNP). Also, $X$ and $X^{*}$ have the same linear dimension and cardinality. If $X$ has Čech complete ball, then ( $X^{* *}$, weak) is realcompact, 
but $X^{* *}$ may fail the Pettis integral property. Moreover, $\left(B_{X^{* *}}\right.$, weak $\left.{ }^{*}\right)$ and $\left(B_{X^{*}}\right.$, weak*) are Eberlein compacts. The dual of a space with Čech complete ball cannot contain $c_{0}$, but may contain $l^{1}$. For spaces $X$ with Čech complete ball, these conditions are equivalent: $X^{*} \not \supset l^{1}, X^{* *}$ has property $(\mathrm{C}),\left(B_{X^{* * *}}\right.$, weak $\left.{ }^{*}\right)$ is angelic.

Here is an indication of the organization of this paper. Section 2 reviews terminology and results from Banach space theory and general topology. Figure 1 may be helpful in relating the various concepts. Section 3 presents the proofs of Theorems A and B. Theorem A can be derived from results of Hurewicz ([28], [53, p. 333]) and Saint-Raymond [49] in a more general setting. We give a self-contained proof. Theorem $\mathrm{B}$ builds on results due to Amir and Lindenstrauss [2] and Valdivia [54]. Section 4 gives the basic structural results about spaces with Čech complete ball. It includes a discussion of properties stronger than Čech completeness. For example, the condition " $B_{X^{* *}} \backslash B_{X}$ is a countable union of weak* compact convex sets" implies both Čech complete ball and the Radon-Nikodym property. Section 5 considers property (PC) in detail. Some partial threespace properties are proved. In particular, if $Y \subseteq X, Y$ has Polish ball, $X / Y$ has property (PC), and $Y^{\perp}$ is complemented in $X^{*}$, then $X$ has property (PC). Section 6 contains a number of examples. Table 1 summarizes information about them.

Thanks are due to $\mathrm{M}$. Talagrand for some helpful conversations. The second author expresses his appreciation to the Department of Mathematics of The Ohio State University for its hospitality during the time that this research was carried out.

2. Preliminary results. Notation and terminology used here can be found, for example, in [39]. A good reference for the point-set topology we shall need is [18].

If $X$ is a Banach space, we write $X^{*}$ for the dual space and $B_{X}$ for the closed unit ball of $X$. We use brackets $\langle$,$\rangle for the pairing between a$ Banach space and its dual: if $x \in X$ and $x^{*} \in X^{*}$, then $\left\langle x, x^{*}\right\rangle=x^{*}(x)$. The space $X$ is identified with a subspace of $X^{* *}$ via the map $j: X \rightarrow X^{* *}$ defined by

$$
\left\langle x^{*}, j(x)\right\rangle=\left\langle x, x^{*}\right\rangle .
$$

In most cases we omit mention of the map $j$. We write ( $X$, weak) for the topological space obtained by equipping the Banach space $X$ with its weak topology ([14, p. 419]). If $A$ is a subset of a Banach space $X$, then its annihilator is

$$
A^{\perp}=\left\{x^{*} \in X^{*}:\left\langle a, x^{*}\right\rangle=0 \text { for all } a \in A\right\} .
$$


If $B$ is a subset of $X^{*}$, then

$$
B_{\perp}=\{x \in X:\langle x, b\rangle=0 \text { for all } b \in B\} .
$$

Occasionally, we will need to refer to more than one topology in the same argument. We write $\operatorname{wcl}(A)$ for the closure of $A$ in the weak topology, and $\mathrm{w}^{*} \mathrm{cl}(A)$ for the closure in the weak* topology of $X^{* *}$. If $X$ is a Banach space, we write $X \hookleftarrow l^{1}$ if $X$ has a closed subspace isomorphic to the space $l^{1}$, and $X \nleftarrow l^{1}$ if not.

In this paper we are primarily concerned with topological properties of the weak topology on a Banach space $X$ or its subsets. Some classical results in this direction are:

(1) $X$ is reflexive if and only if $\left(B_{X}\right.$, weak) is compact, or (equivalently) ( $X$, weak) is $\sigma$-compact $([14, \mathrm{~V} .4 .7])$

(2) If $A$ is a (closed) subset of ( $X$, weak), then $A$ is sequentially compact if and only if $A$ is compact. (Eberlein-Šmulian Theorem, see [14, V. 6.1])

(3) ( $X$, weak) has countable tightness; that is, if $A \subseteq X$ and $x \in$ $\operatorname{wcl}(A)$, then there is a countable set $D \subseteq A$ with $x \in \operatorname{wcl}(D)$. [56, p. 229]

(4) $\left(B_{X}\right.$, weak) is metrizable if and only if $X^{*}$ is separable [14, V. 5.2]. Also ( $B_{X^{*}}$, weak ${ }^{*}$ ) is metrizable if and only if $X$ is separable [14, V. 5.1], so in this case $\left(B_{X^{*}}\right.$, weak $\left.{ }^{*}\right)$ is a compact metric space.

Corson [8] initiated the detailed study of ( $X$, weak). Godefroy [21, 22] used the Čech complete and Polish properties of ( $B_{X}$, weak) in his study of unique isometric preduals. Some of the Banach space ideas used in this paper are discussed in $[\mathbf{1 5}, \mathbf{1 6}]$.

2.1. Definitions. Let $X$ be a Banach space.

(1) The space $X$ is weakly compactly generated (WCG) iff there is a weakly compact subset whose linear span is dense in $X$ (see [37]).

(2) The space $X$ is somewhat reflexive iff every infinite-dimensional closed subspace contains an infinite-dimensional reflexive subspace. If a space is somewhat reflexive, then it cannot contain $c_{0}$ or $l^{1}$. The converse is an important open question.

(3) The space $X$ is an Asplund space iff every separable subspace of $X$ has a separable dual space. It is equivalent to require that $X^{*}$ have the Radon-Nikodým property $[\mathbf{5 0}, \mathbf{5 1}]$. Asplund spaces have a topological characterization: the space $X$ is an Asplund space iff every separable subset of $\left(B_{X}\right.$, weak) is metrizable, or iff every Radon measure on ( $B_{X}$, weak) has metrizable support. 
(4) The space $X$ is a Godefroy space iff (a) $X^{*}$ is separable; (b) $X^{*} \circlearrowleft l^{1}$; and (c) $X^{\perp}$ is weak* separable in $X^{* * *}$. Note that $X^{\perp}=$ $\left(X^{* *} / X\right)^{*}$, and the weak* topology on $X^{\perp}$ in this pairing coincides with the relative weak* topology of $X^{* * *}$.

(5) The space $X$ is separably distinguished iff there is a (norm) separable subspace $Y$ of $X^{*}$ such that if $x^{* *} \in X^{* *}, x \in X, x^{* *}|Y=x| Y$, then $x^{* *} \in X$. It is equivalent to require that $Y^{\perp} \subseteq X^{* *}$ be a subspace of $X$.

(6) The space $X$ has property (PCA) iff $X$ has property (PC) and $X$ is an Asplund space.

(7) The space $X$ has the Radon-Nikodym property (RNP) iff for every non-empty bounded set $A$, and every $\varepsilon>0$, there exists a linear functional $x^{*} \in X^{*}$ and scalar $\alpha$ such that $A \cap\left\{x:\left\langle x, x^{*}\right\rangle>\alpha\right\}$ is non-empty and has diameter less than $\varepsilon$. (Other equivalent definitions are discussed in [13].)

Some of the relationships among these properties are shown in Figure 1. Several examples and counterexamples are discussed in $\S 6$.

Some special properties of the spaces $c_{0}$ and $l^{1}$ will be useful.

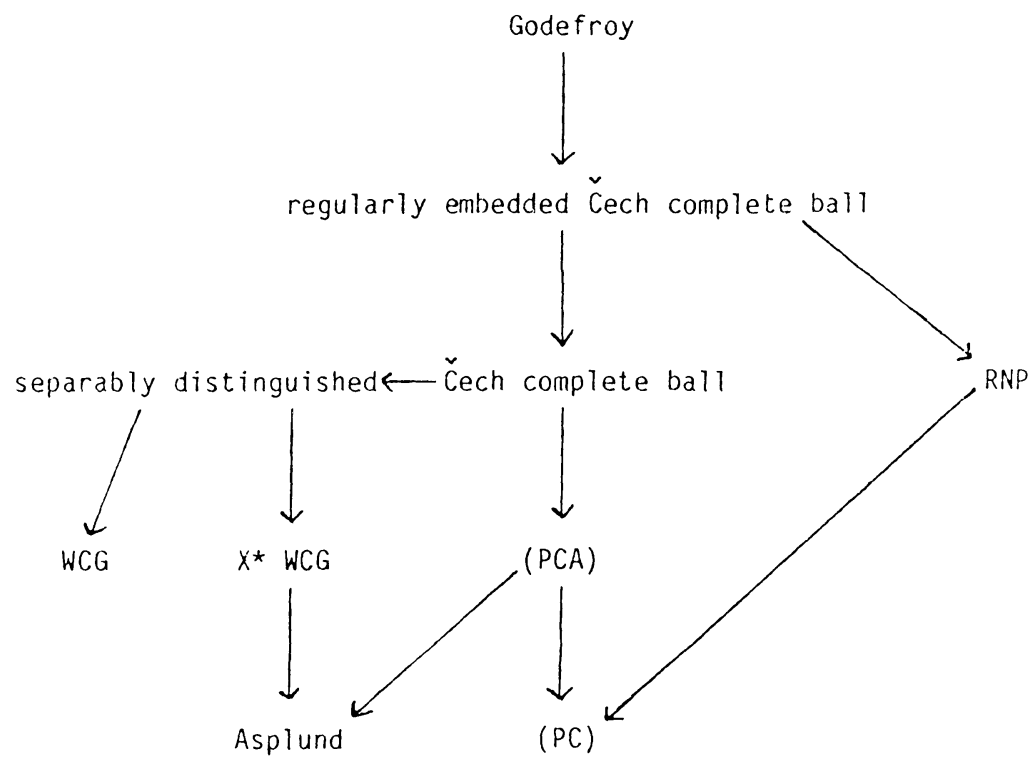

FIGURE 1

2.2. Proposition. (1) If a Banach space $X$ admits a continuous linear map onto $l^{1}(A)$, then $l^{1}(A)$ is isomorphic to a complemented subspace of $X$. (2) If $X^{*} \nleftarrow l^{1}$, then $X \nleftarrow l^{1}$. (3) if $Y$ is a closed subspace of $X$ such that $Y \leftrightarrow l^{1}$ and $X / Y \leftrightarrow l^{1}$, then $X \nleftarrow l^{1}$. (4) If $X$ is separable, then $X^{*} \hookleftarrow l^{1}$ if 
and only if $c_{0}$ is isomorphic to a quotient space of $X$. (5) $X^{*} \hookleftarrow c_{0}$ if and only if $l^{1}$ is isomorphic to a complmented subspace of $X$, and then $l^{\infty}$ is isomorphic to a complemented subspace of $X^{*}$.

For the proof of (1), see [39, p. 107]; (2) follows from (1). For (3), see $[12$, p. 42$]$; (4) is [39, 2.e.9]; (5) is [39, 2.e.8].

Čech completeness for a completely regular Hausdorff space $T$ was defined in the Introduction. In the definition we may take each of the open covers $\mathscr{U}_{n}$ to be a base for the topology of $T$ and to be closed under finite unions $[19,20]$.

A pseudobase for a topological space $T$ is a collection $\mathscr{P}$ of non-empty open sets such that every non-empty open set contains a member of $\mathscr{P}$. The space $T$ is almost $\breve{C}$ ech complete iff it admits a complete sequence of pseudobases. A space is almost Čech complete if and only if it has a dense $G_{\delta}$ subset that is Čech complete [1, p. 28]. Every almost Čech complete space is a Baire space [1].

A norm on a Banach space $X$ is called a Kadec norm iff the weak and norm topologies coincide on $S_{X}=\{x:\|x\|=1\}$. Now $S_{X}$ is dense in $\left(B_{X}\right.$, weak) if $X$ is infinite-dimensional. If the norm is a Kadec norm, then ( $S_{X}$, weak) is a Polish space, hence Čech complete, hence a $G_{\delta}$ in $\left(B_{X}\right.$, weak), so that ( $B_{X}$, weak) is almost Čech complete. Every WCG space is locally uniformly convexifiable, hence admits a Kadec norm [11].

Kerstan [34] studied spaces that are Čech complete and Lindelöf. Such spaces are characterized among the completely regular spaces $T$ by each of the following: (1) If $S$ is any compactification of $T$, then $S \backslash T$ is a countable union of compact $G_{\delta}$ subsets of $S$; (2) $T$ admits a perfect map onto a Polish space; (3) $T$ is homeomorphic to a closed subset of $\mathbf{R}^{\mathbf{N}} \times K$, where $K$ is compact. From Theorem B, the Čech complete ball of a Banach space satisfies (3) for an Eberlein compact $K$.

The proof of the following proposition is left to the reader. Note that (2) is clearly preserved by linear homeomorphisms.

2.3. Proposition. The following are equivalent conditions on a Banach space $X:$ : (1) $\left(B_{X}\right.$, weak) is Cech complete; (2) $X$ is a $G_{\delta}$-set in ( $X^{* *}$, weak $\left.^{*}\right)$; (3) $B_{X^{* *}} \backslash B_{X}$ is weak* $\sigma$-compact; (4) $X^{* *} \backslash X$ is weak ${ }^{*} \sigma$-compact.

2.4. Definition. A Banach space $X$ has regularly embedded Čech complete ball iff $B_{X^{* *}} \backslash B_{X}=\bigcup_{n=1}^{\infty} K_{n}$, where each $K_{n}$ is weak* compact convex and $d\left(K_{n}, X\right)>0$. 
Here $d\left(K_{n}, X\right)$ is the distance from $K_{n}$ to $X$, defined by $\inf \{\|y-x\|$ : $\left.y \in K_{n}, x \in X\right\}$. This property is preserved by linear homeomorphisms. Indeed, if $C_{X}$ is the closed unit ball for an equivalent norm on $X$, with $C_{X} \subseteq m B_{X}$, then

$$
C_{X^{* *}} \backslash C_{X}=C_{X^{* *}} \cap\left(m B_{X^{* *}} \backslash m B_{X}\right)=\bigcup_{n=1}^{\infty}\left(C_{X^{* *}} \cap m K_{n}\right),
$$

where each $C_{X^{* *}} \cap m K_{n}$ is weak* compact convex, and has positive distance from $X$ with respect to the new norm. We do not know if Definition 2.4 is equivalent to the same condition with " $d\left(K_{n}, X\right)>0$ " deleted. Note that if $x^{* *}$ is a point in $X^{* *}$ whose distance $r$ from $X$ is not attained, then $\left\{y^{* *}:\left\|y^{* *}-x^{* *}\right\| \leq r\right\}$ is a weak* compact convex set with zero distance from $X$.

2.5. Definitions. Let $K$ be a compact Hausdorff space.

(1) $K$ is an angelic compact (also called a compact Fréchet space) iff the closure of every subset $A$ is the set of limits of sequences from $A$.

(2) $K$ is an Eberlein compact iff $K$ is homeomorphic to a weakly compact subset of a Banach space (see [37]).

(3) $K$ is a Rosenthal compact [23] iff $K$ is homeomorphic to a pointwise compact set of functions of first Baire class on a Polish space.

The Odell-Rosenthal Theorem [43] asserts that if $X$ is a separable Banach space and $X \nleftarrow l^{1}$, then $K=\left(B_{X^{* *}}\right.$, weak*) is a Rosenthal compact with respect to the Polish space $\left(B_{X^{*}}\right.$, weak*). Every compact metric space is both Eberlein and Rosenthal compact, and each of these is angelic. The product of two Eberlein compacts is an Eberlein compact, and the product of two Rosenthal compacts is a Rosenthal compact. While the product of two angelic compacts need not be angelic, we do have the following result (cf. [24, Th. 1(d)]).

2.6. THEOREM. If $K$ is an Eberlein compact and $L$ is a Rosenthal compact, then $K \times L$ is angelic.

Proof. Let $A \subseteq K \times L$ and $p=\left(k_{0}, l_{0}\right) \in \bar{A}$. Let $U$ be an arbitrary closed neighborhood of $k_{0}$ in $K$. Then

$$
p \in \overline{A \cap(U \times L)}, \quad \text { so } \quad l_{0} \in \overline{\pi_{2}(A \cap(U \times L))} .
$$

Since $L$ is angelic, there is a sequence $\left(k_{i}^{U}, l_{i}^{U}\right)$ in $A \cap(U \times L)$ with $l_{i}^{U} \rightarrow l_{0}$. Since $K$ is angelic, we may assume that $k_{l}^{U} \rightarrow z^{U} \in U$. As the closed neighborhood $U$ of $k_{0}$ decreases, we have $z^{U} \rightarrow k_{0}$. Choose a 
sequence $\left(U_{j}\right)$ with $z^{U_{J}} \rightarrow k_{0}$. Now we have $\left(k_{0}, l_{0}\right)$ in the closure of the countable set

$$
B=\left\{\left(k_{i}^{U_{j}}, l_{i}^{U_{j}}\right): i, j=1,2, \ldots\right\} \subseteq A .
$$

Thus $p \in \bar{B} \subseteq \overline{\pi_{1}(B)} \times L$. Now $\overline{\pi_{1}(B)}$ is a separable Eberlein compact, hence compact metrizable [37], hence a Rosenthal compact. Thus $\overline{\pi_{1}(B)}$ $\times L$ is a Rosenthal compact, so angelic. Thus some sequence in $B$ converges to $p$.

3. Polish ball and property (PC). We begin with a proof of Theorem B. This factorization of spaces with Čech complete ball reduces their study to the separable case, where the special properties of Polish spaces can be used to advantage.

3.1. LemMa. If $X$ is a separable Banach space with $\check{C}$ ech complete ball, then $X^{*}$ is separable.

Proof. By Proposition 2.3, we may write $X=\bigcap_{n=1}^{\infty} U_{n}$, where each $U_{n}$ is a weak* open subset of $X^{* *}$. Each $U_{n}$ contains a basic neighborhood of 0 , determined by a finite set $F_{n}$ in $X^{*}$. Since $X$ is separable, $\left(X^{*}\right.$, weak $\left.{ }^{*}\right)$ is also separable. Let $D$ be a countable dense subset of $\left(X^{*}\right.$, weak*), and let $Y$ be the norm closed linear span of $D \cup \cup_{n=1}^{\infty} F_{n}$ in $X^{*}$. The annihilator $Y^{\perp}=\left\{x^{* *} \in X^{* *}:\left\langle y, x^{* *}\right\rangle=0\right.$ for all $\left.y \in Y\right\}$ is a subset of each $U_{n}$, and contains no non-zero member of $X$. Thus $Y^{\perp}=\{0\}$, so $Y=X^{*}$, and $X^{*}$ is separable.

Note that the proof actually shows that if $X$ is a Banach space with Čech complete ball then $X^{*}$ is norm separable if and only if it is weak* separable.

3.2. Corollary. A Banach space has Polish ball if and only if it is separable and has $\breve{C}$ ech complete ball.

Proof. The necessity is obvious. Since a separable metrizable Čech complete space is Polish [18, p. 190], the sufficiency follows from 3.1.

THEOREM B. Let $X$ be a Banach space. The following are equivalent: (1) $X$ has Čech complete ball; (2) $X$ is isomorphic to a direct sum $R \oplus S$, where $R$ is reflexive and $S$ has Polish ball. 
Proof. (2) $\Rightarrow(1)$. Since ( $X$, weak) is topologically isomorphic to ( $R$, weak $) \times(S$, weak $)$, the unit ball $\left(B_{X}\right.$, weak $)$ is homeomorphic to a closed subset of $\left(B_{R}\right.$, weak $) \times\left(B_{S}\right.$, weak $)$. The topological property of Čech completeness is preserved by closed subsets and countable products $[\mathbf{1}, \mathrm{p}$. 14].

$(1) \Rightarrow(2)$. Choose $\left(U_{n}\right)$ and $\left(F_{n}\right)$ as in 3.1, and let $G$ be the closed linear span of $\bigcup_{n=1}^{\infty} F_{n}$ in $X^{*}$. Then $G^{\perp}$ is a weak* closed subspace of $X^{* *}$, and $G^{\perp} \subseteq \bigcap_{n=1}^{\infty} U_{n}=X$. Since the ball of $G^{\perp}$ is $\sigma\left(X^{* *}, X^{*}\right)$-compact, this makes $R_{1}=G^{\perp}$ a reflexive subspace of $X$. Since $\left(X / R_{1}\right)^{*}=G$ is separable, $X / R_{1}$ is separable. Let $S_{1}$ be a separable subspace of $X$ that maps onto $X / R_{1}$ under the quotient map. Thus $R_{1}+S_{1}=X$. Now $X$ is WCG, since $X$ is the closed span of $B_{R_{1}}+A$, where $A$ is a sequence converging to 0 in $S_{1}$ with span dense in $S_{1}$. By a theorem of Amir and Lindenstrauss [2], there is a separable subspace $S$ of $X$ such that $S_{1} \subseteq S$ and $S$ is complemented in $X$. Hence $X$ is isomorphic to $R \oplus S$, where $R=X / S=$ $\left(X / S_{1}\right) /\left(S / S_{1}\right)$. But $X / S_{1}$ is a quotient of $R_{1}$, so $R$ is a quotient of $R_{1}$, and thus $R$ is reflexive. Finally, $S$ is separable and has Čech complete ball, so it has Polish ball by 3.2 .

Note that Theorem B shows that a space $X$ with $\breve{C e c h}$ complete ball is WCG. Also, $X^{*}=R^{*} \oplus S^{*}$, where $R^{*}$ is reflexive and $S^{*}$ is separable, so $X^{*}$ is WCG.

3.3. ExAmple. The space $c_{0}$ has separable dual, but its ball is not Polish. Indeed, the ball of $c_{0}$ is not a Baire space in the weak topology: it can be expressed as $\bigcup_{n=1}^{\infty} A_{n}$, where each

$$
A_{n}=\left\{x:\left|x_{k}\right| \leq 1 / 2 \text { for all } k \geq n\right\}
$$

is closed and nowhere dense. But if $c_{0}$ is renormed using Day's norm [11, p. 94], which is locally uniformly convex, then the unit sphere is a dense $G_{\delta}$ Polish subspace of the ball, so the ball is a Baire space.

The following result is due to Godefroy.

\subsection{Theorem. A Godefroy space has Polish ball.}

Proof. Recall that a Godefroy space $X$ has these three properties: $X^{*}$ is separable, $X^{*} \nleftarrow l^{1}, X^{\perp}$ is weak* separable. Let $\left(y_{n}\right)$ be a countable dense subset of $\left(X^{\perp}\right.$, weak ${ }^{*}$. Since $X^{*}$ is separable and $X^{*} \leftrightarrow l_{1}$, by the Odell-Rosenthal Theorem [43], each $y_{n}$ is the weak* limit of a sequence 
$\left(x_{n k}^{*}\right)$ in $X^{*}$. Then

$$
\begin{aligned}
X & =\left\{x^{* *}:\left\langle x^{* *}, y_{n}\right\rangle=0 \text { for all } n\right\} \\
& =\bigcap_{n=1}^{\infty} \bigcap_{m=1}^{\infty} \bigcup_{k=m}^{\infty}\left\{x^{* *}:\left|\left\langle x_{n k}^{*}, x^{* *}\right\rangle\right|<\frac{1}{m}\right\}
\end{aligned}
$$

is a weak* $G_{\delta}$ set in $X^{* *}$. By Corollary 3.2, $X$ has Polish ball.

In particular, every Banach space with separable second dual has Polish ball [22]. This holds for separable quasi-reflexive spaces and the separable long James spaces $J(\alpha), \alpha$ countable [17].

An examination of this proof may suggest the following generalization.

3.5. Definition. A Banach space has property (A) iff there exist norm one vectors $y_{n l}^{*}$ in $X^{*}\left(1 \leq i \leq m_{n}, n \in \mathbf{N}\right)$ such that if we define

$$
p_{n}\left(x^{* *}\right)=\max \left\{\left|\left\langle y_{n i}, x^{* *}\right\rangle\right|: 1 \leq i \leq m_{n}\right\},
$$

then (1) $\lim p_{n}(x)=0$ for all $x \in X$, but (2) $\liminf p_{n}\left(x^{* *}\right)>0$ for all $x^{* *} \in X^{* *} \backslash X$.

Any Banach space with property (A) has Čech complete ball, since

$$
X=\bigcap_{k=1}^{\infty} \bigcup_{n=k}^{\infty}\left\{x^{* *}: p_{n}\left(x^{* *}\right)<\frac{1}{k}\right\}
$$

We do not know if every space with Čech complete ball has property (A). Theorem 4.14 is a step in this direction.

3.6. ExAmple. The predual $B$ of the James Tree space JT satisfies property (A), hence has Polish ball. (Basic material on the space JT is in [30], [38].) Note that $B$ is not a Godefroy space, since $B^{\perp}$ is a nonseparable Hilbert space [38, Theorem 1], and not weak* separable. The $y_{n t}^{*}$ can be taken to be the usual boundedly complete basis in JT, called $\left\{e_{n i}\right.$ : $\left.1 \leq i \leq 2^{n}, n \in \mathbf{N}\right\}$ in [38]. The bi-orthogonal functionals $\left\{f_{n l}\right\}$ form a shrinking basis for $B$, and a weak* basis for $\mathrm{JT}^{*}=B^{* *}$. If $x \in B$, then

$$
\lim _{n} \max _{1 \leq l \leq 2^{n}}\left|\left\langle x, e_{n i}\right\rangle\right|=0
$$

(see Lemma 1 of [38]). If $x^{* *} \in \mathrm{JT}^{*} \backslash B$, then $\lim _{n}\left|\left\langle e_{n i_{n}}, x^{* *}\right\rangle\right| \geq \delta>0$ for some branch $\left\{\left(n, i_{n}\right): n \in \mathbf{N}\right\}$ of the binary tree, so

$$
\liminf _{n} \max _{1 \leq i \leq 2_{n}}\left|\left\langle e_{n l}, x^{* *}\right\rangle\right| \geq \delta>0 .
$$

The space $B$ has Polish ball, but fails the Radon-Nikodým property [38, Corollary 4], and is therefore not isomorphic to a dual space. The ball 
of JT is not Polish, since JT has non-separable dual. The space JT* does not have Čech complete ball, since it is not WCG. Since $B \subseteq \mathrm{JT}^{*}$ and $\mathrm{JT}^{*} / B$ both have Čech complete ball, this shows that there is no three space property for Čech completeness of the ball. This proof that $B$ has Polish ball is formally a strengthening of the fact that $B$ has property (PC), which was asserted in [6].

Čech complete ball implies property (PC); the converse holds for spaces with separable dual. The proof centers on the notion of a huskable set (in French, épluchable [21], [22]). It is convenient to state the definition in some generality.

3.7. Definition. Let $X$ be a Banach space, and let $\tau$ be a locally convex topology on $X$ that is coarser than the norm topology. A subset $A$ of $X$ is $\tau$-huskable iff for every $\tau$-open set $U$ with $U \cap A \neq \varnothing$ and every $\varepsilon>0$, there is a $\tau$-open set $V$ with $\varnothing \neq V \cap A \subseteq U \cap A$ and $\operatorname{diam}(V \cap A)=\sup \{\|x-y\|: x, y \in V \cap A\}<\varepsilon$.

Another way to say this is: for each $\varepsilon>0$, the collection of nonempty, relatively $\tau$-open subsets of $A$ with diameter less than $\varepsilon$ is a pseudobase for $(A, \tau)$. In practice, $\tau$ will be either the weak topology on $X$ or the weak* topology on a dual space. Kenderov [33] showed that a dual space has the RNP if and only if each weak* closed bounded set is weak* huskable. The term "huskable", unmodified, shall mean huskable in the weak topology.

3.8. Proposition. Let $A$ be a subset of a Banach space. Then the following are equivalent. (1) $A$ is huskable; (2) $\operatorname{wcl}(A)$ is huskable; (3) $\mathrm{w}^{*} \mathrm{cl}(A)$ is weak* huskable in $X^{* *}$.

Proof. (1) $\Rightarrow(3)$. Let $U \subseteq \mathrm{w}^{*} \mathrm{cl}(A)$ be a non-empty relatively weak* open set, and let $\varepsilon>0$. Then $A$ contains a non-empty, relatively weakly open set $V$ with diameter less than $\varepsilon / 2$ such that $V \subseteq U \cap A$. Choose a relatively weak* open set $W$ in $\mathrm{w}^{*} \operatorname{cl}(A)$ with $W \subseteq U$ and $W \cap A=V$. Since $V$ is weak ${ }^{*}$ dense in $W, \operatorname{diam}(W) \leq \varepsilon / 2<\varepsilon$.

3.9. Proposition. Let $A$ be a norm-closed subset of $X$. Then $A$ is $\tau$-huskable if and only if the identity map $(A, \tau) \rightarrow(A$, norm $)$ is continuous at each point of a $\tau$-dense $\tau-G_{\delta}$ subset of $A$.

Proof. Assume A is $\tau$-huskable, and let $U_{n}=\bigcup\{U \subseteq A$ : $U$ is relatively $\tau$-open in $A$, and $\operatorname{diam}(U)<1 / n\}$. Then $\cap_{n=1}^{\infty} U_{n}$ is exactly the set of 
points of continuity of the identity. We need to show that this set is $\tau$-dense in $A$. Let $V$ be a non-empty, relatively $\tau$-open subset of $A$. Choose recursively a sequence $\left(W_{n}\right)$ of relatively $\tau$-open subsets of $V$ with $\operatorname{diam}\left(W_{n}\right)<1 / n$ and the $\tau$-closure of $W_{n}$ in $A$ contained in $W_{n-1}$ for each $n$. Since $A$ is norm closed, the unique point in $\cap W_{n}$ is a member of $V \cap\left(\cap U_{n}\right)$. This shows $\cap U_{n}$ is dense in $A$.

The converse is left to the reader.

3.10. Proposition. Let $A$ be a weakly closed and bounded subset of $X$. Then $(1) \Rightarrow(2 a) \Leftrightarrow(2 b) \Leftrightarrow(2 c) \Rightarrow(3)$. If $A$ is separable, then all five conditions are equivalent.

(1) $A$ is huskable;

(2a) $(A$, weak) is almost Čech complete;

(2b) $A$ contains a dense $G_{\delta}$ subset of $\mathrm{w}^{*} \mathrm{cl}(A)$;

(2c) $A$ is residual in $\mathrm{w}^{*} \mathrm{cl}(A)$;

(3) $A$ is a Baire space.

Proof. (1) $\Rightarrow(2 \mathrm{a}): \mathscr{U}_{n}=\{U \subseteq A: U$ is relatively open and $\operatorname{diam}(U)<$ $1 / n\}$ is a complete sequence of pseudobases of $A$. (2a) $\Rightarrow(2 \mathrm{~b}): A$ has a dense Čech complete subspace [20], which is necessarily a $G_{\delta}$ in its compactification $\mathrm{w}^{*} \mathrm{cl}(A) .(2 \mathrm{~b}) \Rightarrow(2 \mathrm{c})$ : Obvious. (Residual = comeager means that $\mathrm{w}^{*} \mathrm{cl}(A) \backslash A$ is first category in $\mathrm{w}^{*} \mathrm{cl}(A)$.) (2c) $\Rightarrow(2 \mathrm{a}): A$ contains $B=\cap U_{n}$, where each $U_{n}$ is a dense open subset of $\mathrm{w}^{*} \mathrm{cl}(A)$. Then $B$ is a dense $G_{\delta}$ in $\mathrm{w}^{*} \mathrm{cl}(A)$, hence a dense $G_{\delta}$ Čech complete subset of $A$. (2a) $\Rightarrow(3)$ : This holds in general topology [1, p. 28].

Now assume $A$ is separable in the weak topology. Then the normclosed linear span $Y$ of $A$ is norm-separable, so every subset of ( $A$, norm) has the Lindelöf property. Let $U$ be a non-empty, relatively open subset of ( $A$, weak) and let $\varepsilon>0$. Then $U$ is a countable union of norm-closed balls of radius less than $\varepsilon$, intersected with $U$. Since ( $U$, weak) is a Baire space, one of these weakly closed subsets of $U$ must have a weak interior point (for the relative topology of $U$ of $A$ ). This proves that $A$ is huskable.

The following is motivated by a similar result concerning dentability [40].

3.11. Proposition. A subset $A$ of $X$ is huskable if each countable subset is huskable.

Proof. If $A$ is not huskable, then there is a non-empty relatively weakly open subset $U$ of $A$ and $\varepsilon>0$ such that each non-empty relatively 
weakly open subset of $U$ has diameter $\geq 2 \varepsilon$. Choose any $x_{0} \in U$. Then $x_{0}$ is in the weak closure of $\left\{x \in U:\left\|x-x_{0}\right\| \geq \varepsilon\right\}$. By the countable tightness of the weak topology [56, p. 229], there is a countable subset $C_{1}$ of $\left\{x \in U:\left\|x-x_{0}\right\| \geq \varepsilon\right\}$ with $x_{0} \in \operatorname{wcl}\left(C_{1}\right)$. Next, for each point $x_{1 n} \in$ $C_{1}$ there is a countable subset $C_{2 n}$ of $\left\{x \in U:\left\|x-x_{1 n}\right\| \geq \varepsilon\right\}$ with $x_{1 n} \in \operatorname{wcl} C_{2 n}$; let $C_{2}=U C_{2 n}$. Continue in this way, and let $C=\left\{x_{0}\right\} \cup$ $\cup C_{n}$. Then $C$ is a countable subset of $A$ that is not huskable.

3.12. Corollary. Let $A$ be a weakly closed and bounded subset of $X$. Then $A$ is huskable if every weakly closed separable subset is a Baire space.

Proof. This follows from 3.8, 3.10, and 3.11.

The converses to 3.11 and 3.12 are not true. If $X$ is $c_{0}$ with Day's norm (Example 3.3), then $A=B_{X}$ is huskable by 3.10, but no multiple of the usual ball $U$ of $c_{0}$ is Baire, so some countable subset of $U$ is not huskable.

3.13. TheOREM. Let $X$ be a Banach space. Then the following are equivalent:

(1) X has property (PC);

(2) Every weakly closed bounded subset of $X$ is huskable;

(3) Every weakly closed bounded subset of $X$ is almost Čech complete;

(4) Every weakly closed bounded subset of $X$ is a Baire space. Moreover, it is sufficient that every separable subspace have (PC), or that every weakly closed obunded separable subset have property (2), (3), or (4).

Proof. The equivalence of (2), (3), (4) and their separable variants follows from 3.10 and 3.12. Proposition 3.9 shows that $(2) \Rightarrow(1)$. Finally, the (separable) version of (1) implies the (separable) version of (2), since if $A \subseteq X$ has no relatively weakly open subsets with diameter $<\varepsilon$, then the identity $(A$, weak $) \rightarrow(A$, norm $)$ has no points of continuity.

3.14. Corollary. Banach spaces with Čech complete ball and Banach spaces with the RNP have property (PC).

Proof. If $X$ has the RNP, then every bounded subset is dentable [13, p. 136], and therefore every bounded subset is huskable. If $X$ has Čech complete ball, then every weakly closed bounded subset is Čech complete, hence a Baire space. 
The properties (PC) and RNP are "separably determined". However, $J\left(\omega_{1}\right)$ and $\mathrm{JT}^{*}$ (see §6) do not have Čech complete balls but every separable subspace has Čech complete ball. The predual $B$ of JT has Polish ball, but fails the RNP. The space $l^{1}$ has the RNP, so that the unit ball is huskable for every renorming, but the ball is not Čech complete (since the dual of $l^{1}$ is not separable, see Lemma 3.1). The space JT* has (PC), but does not have Čech complete ball, and fails the RNP.

The next few results establish the equivalence of (PC) and Polish ball for spaces with separable dual. This leads directly to Theorem A.

3.15. Definition. A Banach space $X$ is quasi-Čech complete iff $X=\bigcap_{n=1}^{\infty}\left(F_{n} \cup U_{n}\right)$, where $F_{n}$ is closed and $U_{n}$ is open in $\left(X^{* *}\right.$, weak ${ }^{*}$.

Of course, if $X^{*}$ is separable, then $\left(B_{X^{* *}}\right.$, weak $\left.{ }^{*}\right)$ is metrizable, so a set of the form $\bigcap_{n=1}^{\infty}\left(F_{n} \cup U_{n}\right)$ is a $G_{\delta}$-set. Assertion (3) of the following shows that quasi-Čech completeness is preserved by linear homeomorphisms.

3.16. Proposition. The following conditions on a Banach space $X$ are equivalent: (1) $X$ is quasi-Čech complete; (2) $\left(X^{* *} \backslash X\right.$, weak $\left.{ }^{*}\right)$ is $\sigma$-locally compact; (3) If $A$ is any weakly closed bounded subset of $X$, then $A=$ $\bigcap_{n=1}^{\infty}\left(F_{n} \cup U_{n}\right)$, where $F_{n}$ is compact and $U_{n}$ is open in $\mathrm{w}^{*} \mathrm{cl}(A)$; (4) If $A$ is any weakly closed bounded subset of $X$, then $\mathrm{w}^{*} \mathrm{cl}(A) \backslash A$ is weak ${ }^{*} \sigma$-locally compact.

Proof. To see that $(1) \Leftrightarrow(2)$, note that locally compact spaces are precisely the open subsets of compact spaces. Thus if $X$ is quasi-Čech complete, then $X=\bigcap_{n=1}^{\infty}\left(F_{n} \cup U_{n}\right)$, so that

$$
X^{* *} \backslash X=\bigcup_{n=1}^{\infty} \bigcup_{k=1}^{\infty}\left(\left(X^{* *} \backslash F_{n}\right) \cap\left(k B_{X^{* *}} \backslash U_{n}\right)\right)
$$

is $\sigma$-locally compact. The argument is reversible. The proof that $(3) \Leftrightarrow(4)$ is similar. For $(2) \Rightarrow(4)$, observe that $\mathrm{w}^{*} \mathrm{cl}(A) \backslash A$ is a closed subset of $X^{* *} \backslash X$. For (4) $\Rightarrow(2)$, consider $A=B_{X}$.

3.17. Proposition. For Banach spaces, Čech complete ball $\Rightarrow$ quasiČech complete $\Rightarrow(\mathrm{PC})$.

Proof. The first implication is clear. If $X$ is quasi-Čech complete, and $A$ is a weakly closed bounded subset of $X$, then (by 3.16 (3))

$$
A=\bigcap_{n=1}^{\infty}\left(F_{n} \cup U_{n}\right)
$$


By adjoining the interior of $F_{n}$ to $U_{n}$, we may assume that each $U_{n}$ is weak* dense in $\mathrm{w}^{*} \mathrm{cl}(A)$. Thus $A$ is residual in $\mathrm{w}^{*} \mathrm{cl}(A)$. Now 3.10 and 3.13 show that $X$ has property (PC).

The next result is the main step in the proof of Theorem A. Part (2) can be deduced from a theorem of Hurewicz [28] on analytic sets. We include here a self-contained proof.

3.18. TheOREM (1) If $X$ is separable and has (PC), then $X$ is quasi-ČCech complete; (2) If $X^{*}$ is separable and $X$ has $(\mathrm{PC})$, then $B_{X}$ is $\check{C}$ ech complete.

Proof. (1) Let $A \subseteq X$ be a non-empty, weakly closed, bounded set (such as $B_{X}$ ). Fix $\varepsilon>0$. We define recursively a transfinite sequence $A_{\alpha}$ of closed subsets of $A$, and a sequence $U_{\alpha}$ of open sets in ( $X^{* *}$, weak*). Let $A_{0}=A$. Suppose $A_{\alpha}$ has been defined, and is non-empty. Let $\mathscr{U}_{\alpha}=\{V \subseteq$ $X^{* *}: V$ is weak* open, $V \cap A_{\alpha} \neq \varnothing$, and $\left.\operatorname{diam}\left(V \cap A_{\alpha}\right)<\varepsilon\right\}$. Since $X$ has (PC), $\mathscr{U}_{\alpha}$ is a non-empty collection. Let $U_{\alpha}=\cup \mathscr{U}_{\alpha}$. Then $A_{\alpha+1}=A_{\alpha}$ $\backslash U_{\alpha}$ is a closed proper subset of $A_{\alpha}$. If $\lambda$ is a limit ordinal, let $A_{\lambda}=$ $\cap_{\alpha<\lambda} A_{\alpha}$.

We claim that $A_{\gamma}=\varnothing$ for some countable ordinal $\gamma$. If not, $\left(A_{\alpha}\right)_{\alpha<\omega_{1}}$ is a strictly decreasing sequence of weakly closed sets. Since $X$ is separable, every subset of ( $X$, weak) is Lindelöf, so

$$
A \backslash \bigcap_{\alpha<\omega_{1}} A_{\alpha}=\bigcup_{\alpha<\omega_{1}}\left(A \backslash A_{\alpha}\right)=\bigcup_{\alpha<\gamma}\left(A \backslash A_{\gamma}\right)
$$

for some $\gamma<\omega_{1}$, which contradicts the strictly decreasing property of the $A_{\alpha}$. Hence there is a least $\gamma$ such that $A_{\gamma}=\varnothing$.

Let $B_{\alpha}=\mathrm{w}^{*} \operatorname{cl}\left(A_{\alpha}\right), C_{\alpha}=B_{\alpha} \cup\left(\cup_{\beta<\alpha} U_{\beta}\right)$ for each $\alpha \leq \gamma$. Then

$$
A \subseteq \bigcap_{\alpha \leq \gamma} C_{\alpha} \subseteq C_{0}=\mathrm{w}^{*} \operatorname{cl}(A) \text {. }
$$

We claim that if $x^{* *} \in \bigcap_{\alpha \leq \gamma} C_{\alpha}$, then $d\left(x^{* *}, A\right) \leq \varepsilon$. If $x^{* *} \in B_{\alpha} \cap U_{\alpha}$ for some $\alpha \leq \gamma$, then certainly $d\left(x^{* *}, A\right) \leq \varepsilon$, since there is $V \in \mathscr{U}_{\alpha}$ with $x^{* *} \in B_{\alpha} \cap V \subseteq \mathrm{w}^{*} \operatorname{cl}\left(A_{\alpha} \cap V\right)$, and $\operatorname{diam}\left(A_{\alpha} \cap V\right)<\varepsilon$. But if $x^{* *} \in$ $\bigcap_{\alpha \leq \gamma} C_{\alpha}$, then $x^{* *} \in B_{0}$. But $x^{* *} \notin B_{\gamma}$, so there is a least $\alpha_{0}$ such that $x^{* *} \notin B_{\alpha_{0}}$. Since $x^{* *} \in C_{\alpha_{0}}$, we have $x^{* *} \in U_{\beta}$ for some $\beta<\alpha_{0}$. Thus $x^{* *} \in B_{\beta} \cap U_{\beta}$. So $d\left(x^{* *}, A\right) \leq \varepsilon$, as claimed.

Since $A$ is weakly closed in $X$, it is norm closed in $X^{* *}$. If we take $\varepsilon=1 / n$ in the preceding discussion, we obtain $\left(C_{\alpha, n}\right)_{\alpha<\gamma_{n}}$, and so

$$
A=\bigcap_{n=1}^{\infty} \bigcap_{\alpha \leq \gamma_{n}} C_{\alpha, n} .
$$


Each $C_{\alpha, n}$ is the union of a closed set and an open set in $\left(X^{* *}\right.$, weak*). So $X$ is quasi-Čech complete.

(2) If $X^{*}$ is separable, then $X$ is separable, so the preceding construction can be carried out. But $\left(B_{X^{* *}}\right.$, weak $\left.{ }^{*}\right)$ is metrizable, so the weak* compact sets $B_{\alpha}$ are $G_{\delta}$ sets in $\mathrm{w}^{*} \mathrm{cl}(A)$. Thus each $C_{\alpha}$ is a $G_{\delta}$, so $A$ is a $G_{\delta}$, and thus $A$ is Čech complete.

The significance of quasi-Čech completeness lies in the fact that (for separable $X$ ) it gives a one-set criterion for property (PC), as opposed to conditions on all closed bounded sets.

The proof of 3.18 yields an open cover characterization of property (PC) that is valid even for nonseparable spaces.

3.19. COROllary. A Banach space $X$ has property (PC) if and only if there is a sequence $\left\{V_{\alpha, n}: \alpha \leq \gamma_{n}\right\}_{n \in \mathrm{N}}$ of transfinite open covers of ( $B_{X}$, weak) such that

$$
\operatorname{daim}\left(B_{X} \cap\left(V_{\alpha, n} \backslash \bigcup_{\beta<\alpha} V_{\beta, n}\right)\right)<\frac{1}{n}
$$

for all $\alpha \leq \gamma_{n}$.

Proof. If $X$ has (PC) and $\left\{V_{\beta, n}: \beta<\alpha\right\}$ fails to cover $B_{X}$, then there is a weakly open set $V_{\alpha, n}$ such that $\operatorname{diam}\left(B_{X} \cap\left(V_{\alpha, n} \backslash \bigcup_{\beta<\alpha} V_{\beta, n}\right)\right)<1 / n$. Conversely, if the covers exist, and $A$ is a non-empty weakly closed subset of $B_{X}$, then for each $n$ there is a least $\alpha$ such that $V_{\alpha, n} \cap A \neq \varnothing$. Then $\operatorname{diam}\left(V_{\alpha, n} \cap A\right)<1 / n$.

Theorem A. Let $X$ be a separable Banach space. The following are equivalent.

(1) $\left(B_{X}\right.$, weak $)$ is completely metrizable;

(2) $\left(B_{X}\right.$, weak) is a Polish space;

(3) $X$ has property (PC) and is an Asplund space;

(4) $\left(B_{X}\right.$, weak) is metrizable, and every closed subset of it is a Baire space;

Proof. For separable spaces, (1) and (2) are ways of saying that $X$ has Čech complete ball, using 3.2. Also, (3) and (4) are ways of saying that $X$ has (PC) and $X^{*}$ is separable, using 3.13. Thus the equivalence of (1)-(4) follows from 3.18 . 
Property (3) is intersecting even in nonseparable Banach spaces. If $X$ has property (PC) and $X$ is an Asplund space, we say that $X$ has property (PCA). Equivalently, $X$ has (PCA) if and only if every separable subspace has Polish ball, or every separable subspace has Čech complete ball. Examples of spaces with (PCA) are $J\left(\omega_{1}\right)$ and JT* (see $\S 6$ ).

Another consequence of Theorem $\mathrm{A}$ is that if $X$ is separable, and both $X$ and $X^{*}$ have RNP, then $X$ has Polish ball. The converse is false: the space $B$ of Example 3.6 has Polish ball, but fails the RNP.

4. Čech complete ball. The results of the previous section produce structural information about spaces with Čech complete ball, their duals and their quotients. Here is a refinement of Theorem B.

\subsection{THEOREM. If $X$ has property (PCA), then $X$ is somewhat reflexive.}

Proof. Since every separable subspace of $X$ has Čech complete ball, it suffices to show that every infinite-dimensional space $X$ with Čech complete ball contains an infinite-dimensional reflexive subspace. First, $X^{* *} \backslash X=\bigcup_{n=1}^{\infty} K_{n}$, where each $K_{n}$ is weak* compact. For each $n$, let $\mathscr{U}_{n}=\left\{U \subseteq X: U\right.$ is a weakly open set with $\left.\mathrm{w}^{*} \mathrm{cl}(U) \cap K_{n}=\varnothing\right\}$. Each $\mathscr{U}_{n}$ is an open cover of $\left(X\right.$, weak). Then $\left(\mathscr{U}_{n}\right)_{n=1}^{\infty}$ is a complete sequence of open covers for $B_{X}$. Indeed, if $\mathscr{F}$ is a family of closed subsets of $B_{X}$ with the finite intersection property that is $\mathscr{U}_{n}$ small for each $n$, then $\bigcap_{F \in \mathscr{F}} \mathrm{W}^{*} \mathrm{cl}(F)$ is a non-empty subset of $X^{* *}$ disjoint from all $K_{n}$, so $\bigcap_{F \in \mathscr{F}} F \neq \varnothing$.

We proceed with a recursive construction. Choose $x_{1} \in X, x_{1} \neq 0$, and let $P_{1}: X \rightarrow\left[x_{1}\right]$ be a projection. (We use brackets [ ] for the span of a finite set.) Then $\overline{P_{1}\left(B_{X}\right)}$ is compact, and covered by basic open sets from $\mathscr{U}_{1}$, so there is a finite subcover $\mathscr{U}_{1}^{\prime}$. Choose a finite set $A_{1}$ of $X^{*}$ that determines $P_{1}$ and the members of $\mathscr{U}_{1}^{\prime}$. Let $F_{1}=\left[x_{1}\right]+\left[A_{1}\right]_{\perp}$; this is a closed subspace of $X$ with finite codimension. Also, $B_{F_{1}}$ is covered by $\mathscr{U}_{1}^{\prime}$, since $\left\langle x-P_{1} x, y\right\rangle=0$ for each $x \in F_{1}$ and $y \in A_{1}$.

Now choose $x_{2} \in\left[A_{1}\right]_{\perp} \backslash\left[x_{1}\right]$; this is possible since $X$ is infinite-dimensional. Let $P_{2}: X \rightarrow\left[x_{1}, x_{2}\right]$ be a projection. Then $\overline{P_{1}\left(B_{X}\right)}$ is covered by a finite subfamily $\mathscr{U}_{2}^{\prime}$ of the basic open sets in $\mathscr{U}_{2}$. Choose a finite subset $A_{2}$ of $X^{*}$ that determines $P_{2}$ and the members of $\mathscr{U}_{2}^{\prime}$. Let $F_{2}=$ $\left[x_{1}, x_{2}\right]+\left[A_{1} \cup A_{2}\right]_{\perp}$. Then $F_{2}$ has finite codimension, and $B_{F_{2}}$ is covered by $\mathscr{U}_{2}^{\prime}$.

Continuing in this way, we obtain a linearly independent sequence $x_{1}, x_{2}, \ldots$ in $X$ and a decreasing sequence $F_{1} \supseteq F_{2} \supseteq \cdots$ of subspaces of $X$ such that $x_{j} \in F_{k}$ for $j \leq k$ and $B_{F_{k}}$ is covered by finitely many basic 
open sets from $\mathscr{U}_{k}$. Let $F=\bigcap_{k=1}^{\infty} F_{k}$. Then $x_{j} \in F$ for all $j$, so $F$ is infinite-dimensional. Also, $B_{F}$ is covered by finitely many sets from $\mathscr{U}_{k}$ for all $k$. It follows that $B_{F}$ is weakly compact: If $\mathscr{F}$ is a filter of closed subsets of $B_{F}$, then $\mathscr{F}$ is $\mathscr{U}_{n}$-small for each $n$, since $\mathscr{U}_{n}$ is closed under finite unions. Hence $\cap \mathscr{F} \neq \varnothing$. Thus $F$ is reflexive.

This theorem generalizes a result of Johnson and Rosenthal [31] stating that if $Y$ embeds in a separable dual and $Y^{*}$ is separable, then $Y$ is somewhat reflexive.

Here is another result related to Theorem B.

4.2. Proposition. Let $X$ be a Banach space and $Y$ a reflexive subspace. Then $X$ has Čech complete ball if and only if $X / Y$ has Čech complete ball.

Proof. We have $(X / Y)^{* *}=X^{* *} / Y^{\perp \perp}=X^{* *} / Y$. If $\pi: X^{* *} \rightarrow$ $X^{* *} / Y$ is the quotient map, then $(X / Y)^{* *} \backslash(X / Y)=\pi\left(X^{* *} \backslash X\right)$. The reflexivity of $Y$ shows that $\pi$ maps $B_{X^{* *}}$ onto $B_{X^{* *} / Y}$, so it maps $B_{X^{* *}} \backslash B_{X}$ onto $B_{X^{* *} / Y} \backslash B_{X / Y}$. The map $\pi$ is $\sigma\left(X^{* *}, X^{*}\right)-\sigma\left(X^{* *} / Y, Y^{\perp}\right)$ continuous. It follows that $B_{X^{* *}} \backslash B_{X}$ is weak ${ }^{*} \sigma$-compact if and only if $B_{X^{* *} / Y} \backslash B_{X / Y}$ is weak* $\sigma$-compact. By Theorem 2.3, $B_{X}$ is Čech complete if and only if $B_{X / Y}$ is Čech complete.

This proposition and Corollary 4.7 are positive results about quotients of spaces with Čech complete ball. However, not all quotients preserve the Cech complete or Polish properties of the ball (see Proposition 4.8 and subsequent remarks). The space $\mathrm{JT}^{*}$ admits a separable subspace $B$ with Polish ball such that $\mathrm{JT}^{*} / B$ is reflexive, but $\mathrm{JT}^{*}$ does not have Čech complete ball (since it is not WCG).

Spaces with Čech complete ball are separately distinguished, according to the next result.

4.3. THEOREM. Let $X$ be a Banach space. The following are equivalent: (1) $X$ is separably distinguished; (2) $X$ is isomorphic to $R \oplus S$, where $R$ is reflexive and $S^{*}$ is separable; (3) There is a non-empty $G_{\delta}$ set in $\left(X^{* *}\right.$, weak) that is contained in $X$.

Proof. (1) $\Rightarrow(2)$. Choose a separable subspace $Y$ of $X^{*}$ such that $Y^{\perp}=\left\{x^{* *}:\left\langle y, x^{* *}\right\rangle=0\right.$ for all $\left.y \in Y\right\}$ is a subspace of $X$. As in the proof of Theorem $\mathrm{B}, R_{1}=Y^{\perp}$ is reflexive, $X / R_{1}$ has separable dual, and $X$ is WCG. Moreover, $Y=\left(X / R_{1}\right)^{*}$ is a separable dual space, so it has 
RNP [13, p. 79]. Also, $X^{*} / Y=R_{1}^{*}$ has RNP, since it is reflexive [13, p. 76]. Thus $X^{*}$ has RNP [15, Theorem 6.3], so $X$ is an Asplund space [51]. The remainder of the argument in Theorem B shows that $X$ is isomorphic to $R \oplus S$, where $R$ is reflexive and $S$ is separable. Since $X$ is an Asplund space, $S^{*}$ is separable.

(2) $\Rightarrow(3)$. Let $\left(f_{n}\right)$ be norm dense in $S^{*}$. Then $A=\bigcap_{n=1}^{\infty} f_{n}^{-1}(0)$ is a weak $^{*} G_{\delta}$ in $X^{* *}$. If $x^{* *} \in A$, then $x^{* *} \in R \oplus\{0\} \subseteq X$.

(3) $\Rightarrow(1)$. Let $U$ be a $G_{\delta}$-set in $\left(X^{* *}\right.$, weak*) with $x_{0} \in U \subseteq X$. Then $V=U-x_{0}$ is a $G_{\delta}$ set and $0 \in V \subseteq X$. So there is a countable subset $C$ of $X^{*}$ such that $C^{\perp} \subseteq V \subseteq X$ (as in the proof of Theorem B). If $Y$ is the closed span of $C$, then $Y^{\perp} \subseteq X$, so $X$ is separably distinguished.

Note that (3) cannot be replaced by $\left(3^{\prime}\right)$ : There is a non-empty $G_{\delta}$ set in ( $B_{X^{* *}}$, weak $\left.{ }^{*}\right)$ that is contained in $B_{X}$. Indeed, the usual norm on $l^{1}$ is a Kadec norm, so the unit sphere of $l^{1}$ is such a $G_{\delta}$, but $l^{1}$ fails (2).

4.4. Corollary. Suppose $X$ is separably distinguished. Then $X$ is WCG and an Asplund space, and $X^{*}$ is WCG. The spaces $\left(B_{X^{*}}\right.$, weak $\left.{ }^{*}\right)$ and $\left(B_{X^{* *}}\right.$, weak $\left.k^{*}\right)$ are Eberlein compacts.

4.5. TheOrem. Let $X$ be a Banach space. Then the following are equivalent: (1) $X^{*}$ has Čech complete ball; (2) $X^{* *} / X$ is separable; (3) $X$ is isomorphic to $R \oplus S$, where $R$ is reflexive and $S^{* *}$ is separable.

Proof. (1) $\Rightarrow(2)$. Since $X^{*}$ has Čech complete ball, the space

$$
X^{* * *} \backslash X^{*}=\left\{x^{*}+x^{\perp}: x^{*} \in X^{*}, x^{\perp} \in X^{\perp}, x^{\perp} \neq 0\right\}
$$

is weak* $\sigma$-compact. Now $X^{\perp}$ is weak* closed, so $X^{\perp} \cap\left(X^{* * *} \backslash X^{*}\right)=$ $X^{\perp} \backslash\{0\}$ is weak* $\sigma$-compact. Thus $\{0\}$ is a weak* $G_{\delta}$ in $X^{\perp}=$ $\left(X^{* *} / X\right)^{*}$, so there is a countable subset $C$ of $X^{* *} / X$ with $C^{\perp}=\{0\}$. Thus the norm closed span of $C$ is $X^{* *} / X$, so $X^{* *} / X$ is separable.

$(2) \Rightarrow(3)$ is a result of Valdivia [54]. Note that $X^{* *} / X$ and $S^{* *} / S$ can be identified.

(3) $\Rightarrow(1)$. Since $S^{*}$ is a separable dual, it has RNP [13, p. 79] so it has (PC). By 3.18, $S^{*}$ has Čech complete ball, so $R^{*} \oplus S^{*}$ has Čech complete ball.

Note the relative strengths of the factorization properties in Theorems $\mathrm{B}, 4.3$, and 4.5. 
4.6. Corollary. Suppose $X$ is separable. Then the following are equivalent: (1) $B_{X^{*}}$ is Čech complete; (2) $B_{X^{*}}$ is Polish; (3) $X^{* *}$ is separable.

4.7. CoROllaRY. If $X^{*}$ has Čech complete ball, then $X$ (and every quotient space of $X$ ) has $\check{C}$ ech complete ball.

Proof. Let $Z=X / Y$ be a quotient of $X$. Then $Z^{* *} / Z$ is separable since $X^{* *} / X$ is separable [41]. So by $4.5, Z=R \oplus S$, where $S^{* *}$ is separable, so $Z$ has Čech complete ball, by 3.4 .

Thus a dual space with Čech complete ball must have the RNP, since it is the dual of an Asplund space (or, alternatively, since it is WCG). The predual $B$ of James Tree has Polish ball, but fails the RNP [38], and its dual JT does not have Čech complete ball (since $\mathrm{JT}^{*}$ is not separable).

Clark [7] proved that if $X^{* *} / X$ is separable, then $X$ and $X^{*}$ are somewhat reflexive (see also [10], [31]). Here 4.5 and 4.7 show that the conclusion can be strengthened to: $X$ and $X^{*}$ have Čech complete ball. Kuo [35] proved that if $X^{* *} / X$ is separable, then $X^{*}$ and $X^{* *}$ have the RNP. This follows from 4.5, which shows that such an $X^{*}$ or $X^{* *}$ is a sum of a reflexive space and a separable dual space.

4.8. EXAMPLE. If $S$ is a separable Banach space, let $X=\operatorname{JL}(S)$ denote the associated James-Lindenstrauss space [29], [36]. In more detail, $X^{*}$ is separable, and admits a quotient map $\pi$ onto $S$. If $j: X \rightarrow X^{* *}$ is the canonical embedding, then $X^{* *}=j(X) \oplus \pi^{*}\left(S^{*}\right)$. The space $Y=\operatorname{ker} \pi$ is a predual of $X$, since $Y^{*}=X^{* *} /(\operatorname{ker} \pi)^{\perp}$. Thus $X$ always has Polish ball (whether or not $S$ does). By 4.5, the space $X^{*}$ has Polish ball if and only if $S^{*}$ is separable.

4.9. Proposition. The quotients of spaces with Polish ball are precisely the spaces with separable dual.

Proof. If $X$ has Polish ball and $\pi$ maps $X$ onto $Y$, then $Y^{*} \subseteq X^{*}$ is separable. Conversely, if $S^{*}$ is separable, then $\mathrm{JL}(S)^{*}$ has Polish ball and has $S$ as a quotient.

Since $c_{0}$ has separable dual, it can be the quotient of a space with Polish ball, so $l^{1}$ can be a subspace of a dual of a space with Polish ball. In fact, Example (2) in $\S 6$ has Polish ball and dual isomorphic to $l^{1}$. 
If $X$ has Čech complete ball (or is even separably distinguished), then ( $X^{* *}$, weak) must be realcompact. Indeed, $X=R \oplus S$, where $S^{*}$ is separable. Thus $\left(S^{* * *}\right.$, weak $)$ is separable, so $\left(S^{* *}\right.$, weak) is realcompact, using Corson's criterion [8, Lemma 9]. Not much more than realcompactness can be expected for $X^{* *}$ where $X$ has Čech complete ball. If $X=\mathrm{JL}\left(l^{1}\right)$ then $X$ has Polish ball, but $l^{\infty}$ is a direct summand of $X^{* *}$, so $X^{* *}$ fails the Pettis integral property. It may be helpful to consult Figure 1 of [16]; M. Talagrand has recently shown that property $(C)$ implies the Pettis integral property.

We have no characterization of the remainders $X^{* *} / X$, for $X$ with Čech complete ball. Any such remainder is a quotient of the dual of a separable space, hence has at most the cardinal of the continuum. If $X^{* *}=X \oplus Y$, where $Y$ has Čech complete ball, then $X$ has Čech complete ball if and only if $Y$ is separable. Indeed, if $Y$ is separable, then $X^{* *} / X$ is separable, so $X^{*}$ has Čech complete ball (4.5), and hence $X$ has Čech complete ball (4.7). Conversely, if $X$ has Čech complete ball, then $X^{* *}=X \oplus Y$ has Čech complete ball, so $X^{*}$ has Čech complete ball (4.7), and hence $Y=X^{* *} / X$ is separable (4.5).

As an example, let $J$ be James' original quasi-reflexive space of order 1. Then an uncountable $l^{2}$ sum of copies of $J$ does not have Čech complete ball. If $X^{*} \nleftarrow l^{1}$, then more can be said about $X^{* *}$ and $X^{* * *}$. A Banach space $X$ has property (C) if any collection of closed, bounded, convex sets with empty intersection has a countable subcollection with empty intersection. See [45], [46].

4.10. TheOREM. Let $X$ be a separably distinguished Banach space. Then the following are equivalent:

(1) $\left(B_{X^{* * *}}\right.$, weak $\left.^{*}\right)$ is angelic;

(2) $\left(B_{X^{\perp}}\right.$, weak $\left.{ }^{*}\right)$ is angelic;

(3) $\left(B_{X^{* * *}}\right.$, weak $\left.{ }^{*}\right)$ is sequentially compact;

(4) $\left(B_{X^{\perp}}\right.$, weak $\left.{ }^{*}\right)$ is sequentially compact;

(5) $X^{* *}$ has property $(\mathrm{C})$;

(6) $X^{* *} / X$ has property $(\mathrm{C})$;

(7) $X^{* *} \nleftarrow l^{1}\left(\aleph_{1}\right)$;

(8) $X^{*} \leftrightarrow l^{1}$.

Proof. First, let $X$ be any Banach space. Then certainly (1) $\Rightarrow(2) \Rightarrow(4)$ and $(1) \Rightarrow(3) \Rightarrow(4)$. The results of Pol $([\mathbf{4 5}]$ or $[\mathbf{4 6}])$ show that $(1) \Rightarrow(5)$ and $(2) \Rightarrow(6)$. Since property $(C)$ is preserved by continuous images, $(5) \Rightarrow(6)$. Since $l^{1}\left(\boldsymbol{\aleph}_{1}\right)$ fails property $(C)$, we have $(5) \Rightarrow(7)$. Also (7) $\Rightarrow(8)$, 
for if $X^{*} \hookleftarrow l^{1}$, then $l^{\infty}$ is a quotient of $X^{* *}$. But $l^{\infty} \hookleftarrow l^{1}\left(\boldsymbol{\aleph}_{1}\right)$, so $l^{1}\left(\boldsymbol{\aleph}_{1}\right)$ is a quotient of some subspace of $X^{* *}$, and thus (by 2.2(1)) we have $X^{* *} \hookleftarrow l^{1}\left(\boldsymbol{\aleph}_{1}\right)$.

Thus it is enough to show $(6) \Rightarrow(5),(4) \Rightarrow(8),(8) \Rightarrow(1)$ for separably distinguished spaces.

(6) $\Rightarrow(5)$. Suppose $X^{* *} / X$ has property (C). Since $X$ is separably distinguished, it is WCG (by 4.4), hence weakly Lindelöf, and thus $X$ has property $(\mathrm{C})$. Then $X^{* *}$ has $(\mathrm{C})$ by the three-space property [45].

(4) $\Rightarrow(8)$. Since $X$ is separably distinguished, $X=R \oplus S$, where $R$ is reflexive and $S^{*}$ is separable (by 4.3). If $X^{*} \hookleftarrow l^{1}$, then $S^{*} \hookleftarrow l^{1}$ by 2.2 (3). Then by $2.2(4)$, there is a continuous linear map $\pi$ from $X$ onto $c_{0}$. Thus $\pi^{* *}$ maps $X^{* *}$ onto $l^{\infty}$ and induces a continuous linear map of $X^{* *} / X$ onto $l^{\infty} / c_{0}$. The adjoint is a weak* continuous injection of $\left(l^{\infty} / c_{0}\right)^{*}$ into $X^{\perp}$. But $\left(\left(l^{\infty} / c_{0}\right)^{*}\right.$, weak $\left.{ }^{*}\right)$ contains a homeomorph of $\beta \mathbf{N} \backslash \mathbf{N}$, which is not sequentially compact.

(8) $\Rightarrow(1)$. Suppose $X^{*} \nleftarrow l^{1}$. As before, $X=R \oplus S$, where $R$ is reflexive and $S^{*}$ separable. So $S^{*}$ is separable and does not contain $l^{1}$. By the Odell-Rosenthal theorem [43], $\left(B_{S^{* * *}}\right.$, weak $\left.{ }^{*}\right)$ is a Rosenthal compact. But $\left(B_{R^{*}}\right.$, weak $\left.{ }^{*}\right)$ is an Eberlein compact, so by Theorem 2.6, $\left(B_{X^{* * *}}\right.$, weak $\left.^{*}\right)$ is angelic.

We close this section by considering properties formally stronger than Čech complete ball. If $X$ has Čech complete ball, then $X^{* *} \backslash X=\bigcup_{n=1}^{\infty} K_{n}$, where each $K_{n}$ is weak* compact. Additional requirements might be that each $K_{n}$ be convex, or have positive distance from $X$ (see Definition 2.4).

4.11. Lemma. Let $X$ be a Banach space. Suppose $X^{*}$ is separable and $X^{*} \leftrightarrow l^{1}$. Then $X$ has regularly embedded $\check{C}$ ech complete ball if and only if $X^{\perp}$ is weak ${ }^{*}$ separable.

Proof. Assume $X$ has regularly embedded Čech complete ball. Then $X^{* *} \backslash X=\bigcup_{n=1}^{\infty} K_{n}$, where each $K_{n}$ is weak* compact, convex, and $d\left(K_{n}, X\right)>\varepsilon_{n}>0$. If $\pi$ is the quotient map from $X^{* *}$ onto $X^{* *} / X$, then $\pi\left(K_{n}\right)$ is convex, and does not contain 0 in its norm closure. Therefore there exists $f_{n} \in B_{X^{\perp}}$ such that $f_{n}\left(\pi\left(x^{* *}\right)\right)>\varepsilon_{n}$ for all $x^{* *} \in K_{n}$. So $X=\left\{x^{* *} \in X^{* *}: f_{n}\left(\pi\left(x^{* *}\right)\right)=0\right.$ for all $\left.n\right\}=\left\{f_{n}: n \in \mathbf{N}\right\}^{\perp}$, so $X^{\perp}=$ $\left\{f_{n}: n \in \mathbf{N}\right\}^{\perp \perp}$ is weak* separable.

Conversely, let $\left(y_{n}\right)$ be a countable dense subset of $\left(X^{\perp}\right.$, weak*). By the Odell-Rosenthal Theorem [43], for each $n$ there is a sequence $\left(x_{n k}^{*}\right)$ in $X^{*}$ that converges to $y_{n}$ in the weak* topology. Then $X^{* *} \backslash X$ is a 
countable union of sets of the form

$$
\begin{aligned}
& \bigcap_{k=m}^{\infty}\left\{x^{* *}:\left|\left\langle x_{n k}^{*}, x^{* *}\right\rangle\right| \geq \frac{1}{m}\right\}, \\
& \bigcap_{k=m}^{\infty}\left\{x^{* *}:\left|\left\langle x_{n k}^{*}, x^{* *}\right\rangle\right| \leq-\frac{1}{m}\right\}
\end{aligned}
$$

(see the proof of 3.4). These sets are weak* closed, convex, and have distance at least $1 /\left(m\left\|y_{n}\right\|\right)$ from $X$. Indeed, if $x^{* *}$ belongs to either of these sets, and $x \in X$, then

$$
\begin{aligned}
\left\|x^{* *}-x\right\|\left\|y_{n}\right\| & \geq\left|\left\langle y_{n}, x^{* *}\right\rangle-\left\langle y_{n}, x\right\rangle\right|=\left|\left\langle y_{n}, x^{* *}\right\rangle\right| \\
& =\lim _{k}\left|\left\langle x_{n k}^{*}, x^{* *}\right\rangle\right| \geq 1 / m .
\end{aligned}
$$

Intersecting these sets with balls $n B_{X^{* *}}$ yields compact sets.

4.12. THEOREM. If either (1) $X$ is a Godefroy space or (2) $X^{*}$ has $\check{C} e c h$ complete ball, then $X$ has regularly embedded $\breve{C}$ ech complete ball.

Proof. If (1) holds, then $X$ has regularly embedded Čech complete ball by 4.11. If (2) holds, then $X$ is isomorphic to $R \oplus S$, where $R$ is reflexive and $S^{* *}$ is separable (by 4.5). Then $S$ is a Godefroy space, so $S^{* *} \backslash S=$ $\bigcup_{n=1}^{\infty} L_{n}$, where each $L_{n}$ is weak* compact, convex, and $d\left(L_{n}, S\right)>0$. Then

$$
X^{* *} \backslash X=R+\left(S^{* *} \backslash S\right)=\bigcup_{m} \bigcup_{n}\left(m B_{R}+L_{n}\right),
$$

which is the required decomposition.

4.13. TheOREM. Suppose $X^{* *} \backslash X=\bigcup_{n=1}^{\infty} K_{n}$, where each $K_{n}$ is weak ${ }^{*}$ compact and convex. Then $X$ has the Radon-Nikodym property.

Proof. First suppose $X$ is separable. Let $(\Omega, \Sigma, \mu)$ be a probability space, and let $F: \Sigma \rightarrow X$ be a vector measure with $\|F(E)\| \leq \mu(E)$ for all $E \in \Sigma$. This measure has a weak* density in $X^{* *}$ (see [13, p. 84]), that is, a weak* measurable function $f: \Omega \rightarrow X^{* *}$ such that $\left\langle F(E), x^{*}\right\rangle=$ $\int_{E}\left\langle x^{*}, f(\omega)\right\rangle d \mu(\omega)$ for all $E \in \Sigma$ and $x^{*} \in X^{*}$. We will show that $f$ has almost all of its values in $X$. Since $X$ has Polish ball, $X^{*}$ is separable, so $K_{n}$ is a weak* Baire set in $X^{* *}$, so $D_{n}=f^{-1}\left(K_{n}\right)$ belongs to $\Sigma[15$, Theorem 2.3]. If $\mu\left(D_{n}\right)>0$ for some $n$, then $F\left(D_{n}\right) / \mu\left(D_{n}\right)$ is in $X$, so not in $K_{n}$, so by the Hahn-Banach theorem there is $x^{*} \in X^{*}$ and $\alpha$ such that 


$$
\begin{gathered}
\left\langle F\left(D_{n}\right) / \mu\left(D_{n}\right), x^{*}\right\rangle>\alpha>\left\langle x^{*}, y^{* *}\right\rangle \text { for all } y^{* *} \in K_{n} \text {. But then } \\
\int_{D_{n}}\left\langle x^{*}, f(\omega)\right\rangle d \mu(\omega) \leq \alpha \mu\left(D_{n}\right)<\left\langle F\left(D_{n}\right), x^{*}\right\rangle,
\end{gathered}
$$

a contradiction. Thus $\mu\left(D_{n}\right)=0$ for all $n$, so $f(\omega) \in X$ for almost all $\omega$. Then $f$ (redefined to be 0 on a $\mu$-null set) is weakly measurable, and is the Pettis derivative of $F$. Since $X$ is separable, $f$ is Bochner integrable. This shows that $X$ has the RNP.

Now consider the nonseparable case. By Theorem B, $X=R \oplus S$, where $R$ is reflexive and $S$ is separable. Then $S^{* *}$ can be identified with $S^{\perp \perp}$ in $X^{* *}$, and $S^{\perp \perp}$ is closed in $\left(X^{* *}\right.$, weak*). If $X^{* *} \backslash X=\bigcup_{n=1}^{\infty} K_{n}$, where each $K_{n}$ is weak* compact and convex, then

$$
S^{* *} \backslash S=\bigcup_{n=1}^{\infty}\left(S^{\perp \perp} \cap K_{n}\right),
$$

where each $S^{* *} \cap K_{n}$ is also weak* compact and convex. By the first part of the argument, $S$ has RNP. Therefore $X=R \oplus S$ also has RNP.

The predual $B$ of the James Tree space has Čech complete ball, but fails the RNP. The weak* compact sets $K_{n}$ in the decomposition of $B^{* *} \backslash B$ can be chosen to have positive distance from $B$, but not to be convex.

Property (A) is defined in 3.5.

4.14. Theorem. Let $X$ be a Banach space. Then $X$ has property (A) if and only if there exist weak ${ }^{*}$ compact sets $K_{n}$ in $X^{* *}$ with $d\left(K_{n}, X\right)>0$ such that $X^{* *} \backslash X=\bigcup_{n=1}^{\infty} K_{n}$.

Proof. Assume $X$ has property (A), that is, there exist norm-one vectors $y_{n i}^{*} \in X^{*}\left(1 \leq i \leq m_{n}, n \in \mathbf{N}\right)$ such that if

$$
p_{n}\left(x^{* *}\right)=\max \left\{\left|\left\langle y_{n i}^{*}, x^{* *}\right\rangle\right|: 1 \leq i \leq m_{n}\right\},
$$

then $\lim p_{n}(x)=0$ for all $x \in X$ but $\liminf p_{n}\left(x^{* *}\right)>0$ for all $x^{* *} \in$ $X^{* *} \backslash X$. Let

$$
L_{k m}=m B_{X^{* *}} \cap\left\{x^{* *}: p_{n}\left(x^{* *}\right) \geq 1 / k \text { for all } n \geq k\right\} .
$$

Then $X^{* *} \backslash X=\bigcup_{k, m} L_{k m}$ and $d\left(L_{k m}, X\right) \geq 1 / k$.

For the converse, suppose first that $X$ is separable. Let $\left(x_{j}\right)$ be dense in $X$ and let $X^{* *} \backslash X=\bigcup_{n=1}^{\infty} K_{n}$, where $d\left(K_{n}, X\right)>\varepsilon_{n}>0$. So if $x^{* *} \in$ $K_{n}$, then $d\left(x^{* *}, X\right)>\varepsilon_{n}$, so there exists $g_{x^{* *}} \in X^{\perp} \subseteq X^{* * *}$ with $\left\|g_{x^{* *}}\right\|=1$ 
and $\left\langle x^{* *}, g_{x^{* *}}\right\rangle>\varepsilon_{n}$. We can find, for $k \in \mathbf{N}$, functionals $g_{x^{* *}, k} \in B_{X^{*}}$ with $\left\langle x_{j}, g_{x^{* *}, k}\right\rangle<1 / k$ for $1 \leq j \leq k$ and $\left\langle x^{* *}, g_{x^{* *}, k}\right\rangle>\varepsilon_{n}$. This shows that, for each $k$,

$$
K_{n} \subseteq \bigcup_{x^{* *} \in K_{n}}\left\{y^{* *}:\left\langle y^{* *}, g_{x^{* *}, k}\right\rangle>\varepsilon_{n}\right\},
$$

so (by compactness of $K_{n}$ ) there is a finite subcover, say

$$
K_{n} \subseteq \bigcup_{i}\left\{y^{* *}:\left\langle y^{* *}, f_{n i k}\right\rangle>\varepsilon_{n}\right\}
$$

where $i$ runs over a finite set $\left\{1,2, \ldots, m_{n k}\right\}$, and $f_{n i k} \in B_{X^{*}},\left\langle x_{j}, f_{n i k}\right\rangle<$ $1 / k$ for $1 \leq j \leq k$, and if $x^{* *} \in K_{n}$, then $\max _{1 \leq i \leq m_{n k}}\left\langle x^{* *}, f_{n i k}\right\rangle>\varepsilon_{n}$ for all $k$. Define

$$
P_{k}\left(x^{* *}\right)=\max \left\{\left|\left\langle x^{* *}, f_{n l k}\right\rangle\right|: 1 \leq n \leq k, 1 \leq i \leq m_{n k}\right\} .
$$

Now if $x^{* *} \notin X$ then $x^{* *} \in K_{n}$ for some $n$, so that $P_{k}\left(x^{* *}\right)>\varepsilon_{n}$ for all $k \geq n$. On the other hand, if $x \in X$ and $\|x\|=1$, then given $\varepsilon>0$ there exists $j$ with $\left\|x-x_{j}\right\|<\varepsilon / 2$. If $k>\max \{j, 2 / \varepsilon\}$, then for $n \leq k$ and $1 \leq i \leq m_{n k}$, we have

$$
\left|\left\langle x, f_{n \imath k}\right\rangle\right| \leq\left\|x-x_{j}\right\|\left\|f_{n i k}\right\|+\left|\left\langle x_{j}, f_{n i k}\right\rangle\right| \leq \varepsilon / 2+1 / k<\varepsilon .
$$

Thus $p_{k}(x) \leq \varepsilon$. This shows that $X$ has property (A).

For the nonseparable case, observe that $X$ has Cech complete ball, so $X=R \oplus S$, where $R$ is reflexive and $S$ has Polish ball. Thus $S^{* *}=S^{\perp \perp}$ is weak* closed in $\left(X^{* *}\right.$, weak*), so $S^{* *} \backslash S=\bigcup_{n=1}^{\infty}\left(S^{\perp \perp} \cap K_{n}\right)$. By the separable case, $S$ has property (A), and it follows that $X$ does too.

5. A factorization theorem for property (PC). The main theorem of this section employs a version of the Principle of Local Reflexivity. We record it here for convenience. A proof is sketched in [32].

5.1. Proposition. Let $X$ be a Banach space, $U$ and $F$ finite-dimensional subspaces of $X^{* *}$ and $X^{*}$ respectively, $\varepsilon>0$. Then there is a one-to-one linear operator $T: U \rightarrow X$ such that (1) $T x=x$ for all $x \in X \cap U$; (2) $\left\langle T\left(x^{* *}\right), x^{*}\right\rangle=\left\langle x^{*}, x^{* *}\right\rangle$ for all $x^{*} \in F, x^{* *} \in U$; and (3) $\|T\|\left\|T^{-1}\right\|<$ $1+\varepsilon$.

Note that if $X \cap U \neq\{0\}$, then (1) implies $\|T\| \geq 1$ and $\left\|T^{-1}\right\| \geq 1$, so by (3) we have $\|T\|<1+\varepsilon,\left\|T^{-1}\right\|<1+\varepsilon$.

5.2. TheOREM. Let $X$ be a Banach space, $Y$ a closed subspace. Suppose (1) $Y$ has Polish ball; (2) $X / Y$ has property (PC); and (3) $Y^{\perp}$ is a complemented subspace of $X^{*}$. Then $X$ has $(\mathrm{PC})$. 
Proof. Let $i: Y \rightarrow X$ be the inclusion and $\pi: X \rightarrow X / Y$ the quotient map. Let $X^{*}=Y^{\perp} \oplus F$, where $F$ is isomorphic to $Y^{*}$. Then there is a linear isomorphism $u: Y^{*} \rightarrow X^{*}$ of $Y^{*}$ onto the closed subspace $F$ of $X^{*}$, so that $i^{*} \circ u$ is the identity on $Y^{*}$. The space $X^{* *}$ is isomorphic to $\left(X^{* *} / Y^{\perp \perp}\right) \oplus Y^{* *}$, with projections $\pi^{* *}$ and $u^{*}$. There is an $\alpha<\infty$ such that

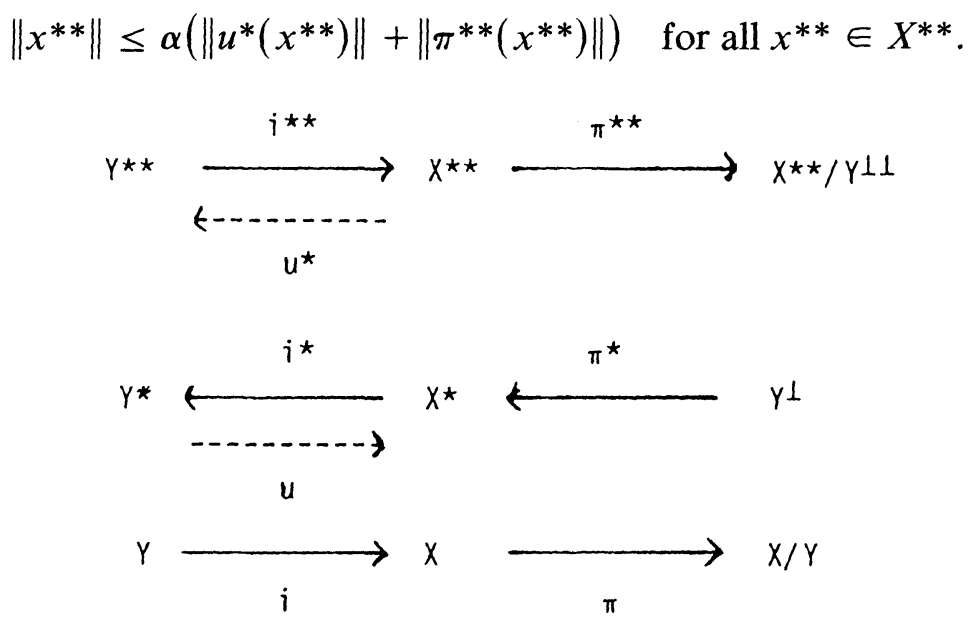

FIGURE 2

Let $A$ be a nonempty bounded subset of $X$. We will show that $A$ contains nonempty relatively weakly open subsets of arbitrarily small diameter. (This will show that $X$ has (PC), by 3.13.) If not, we may assume that $\operatorname{diam}(U \cap A)>1$ for each weakly open set $U$ with $U \cap A \neq \varnothing$. Let $M=1+\operatorname{diam}(A), B=\{y \in Y:\|y\| \leq M\}$. Since $B$ is Polish, there is a complete sequence $\left(\mathscr{U}_{n}\right)$ of open covers of $B$ closed under finite unions. Choose $n_{0}$ such that $2^{n_{0}}>\max \left(2 \alpha, 2,1+2\left\|u^{*}\right\|\right)$.

We will use the following notation for the binary tree $T=\bigcup_{n=0}^{\infty}\{0,1\}^{n}$, and the Cantor set $\Delta=\{0,1\}^{\mathbf{N}}$. If $t \in\{0,1\}^{n}$, its length is $n,|t|=n$. If $k \leq n$, the restriction $t \mid k$ is the element of $\{0,1\}^{k}$ that agrees with $t$ in the first $k$ terms. Similarly, if $\tau \in \Delta$, then $\tau \mid k \in\{0,1\}^{k}$ is an initial segment. For $t \in\{0,1\}^{n}$, its two successors in $\{0,1\}^{n+1}$ are written $t 0$ and $t 1$. We write $\varnothing$ for the element of $\{0,1\}^{0}$.

For each $t \in T$, we will choose vectors $x_{t} \in A, y_{t} \in B$, a basic neighborhood $V_{t}$ of 0 in ( $Y$, weak), and a weakly open subset $W_{t}$ of $X / Y$ such that $\pi\left(x_{t}\right) \in W_{t}$. We do this recursively on $T$.

First, $\pi(A)$ is bounded in $X / Y$, so there is a weakly open set $W_{\varnothing}$ in $X / Y$ with $\pi(A) \cap W_{\varnothing} \neq \varnothing$ and $\operatorname{diam}\left(\pi(A) \cap W_{\varnothing}\right)<2^{-n_{0}}$. Choose $x_{\varnothing} \in$ $A \cap \pi^{-1}\left(W_{\varnothing}\right)$, let $y_{\varnothing}=0$, and let $V_{\varnothing}$ be a basic neighborhood of 0 in $Y$ such that $V_{\varnothing} \cap B$ is contained in an element of $\mathscr{U}_{0}$. 
Now suppose, for some $t \in T$, that $x_{t}, y_{t}, V_{t}, W_{t}$ have been constructed. Let $n=|t|$. Now $V_{t}$ is a basic neighborhood of 0 in $Y$, so there is a finite subset $\left\{f_{j}\right\}_{j=1}^{m}$ of $Y^{*}$ such that $V_{t}=\left\{y:\left|\left\langle y, f_{j}\right\rangle\right|<1,1 \leq j \leq m\right\}$. Let $f_{j}^{\prime}=u\left(f_{j}\right) \in X^{*}$, and let $U_{t}=\left\{x:\left|\left\langle x, f_{j}^{\prime}\right\rangle\right|<1,1 \leq j \leq m\right\}$, a neighborhood of 0 in $X$. Now $X / Y$ has (PC), so $W_{t}$ contains a weakly open set $W_{t 0}=W_{t 1}$ such that $\pi\left(\left(U_{t}+x_{t}\right) \cap A\right) \cap W_{t 0}$ is nonempty and has diameter less than $2^{-\left(n_{0}+n+1\right)}$. Then $\left(\left(U_{t}+x_{t}\right) \cap \pi^{-1}\left(W_{t 0}\right)\right) \cap A$ is nonempty, so it has diameter greater than 1 . Choose $x_{t 0}, x_{t 1}$ in this set with $\left\|x_{t 0}-x_{t 1}\right\|>1$. Note that $\left\|\pi\left(x_{t 0}\right)-\pi\left(x_{t 1}\right)\right\|,\left\|\pi\left(x_{t i}\right)-\pi\left(x_{t}\right)\right\|, i=$ 0,1 , are less than $2^{-\left(n_{0}+n+1\right)}$. Thus

$$
\begin{aligned}
\left\|u^{*}\left(x_{t 0}\right)-u^{*}\left(x_{t 1}\right)\right\| & >\frac{1}{\alpha}\left\|x_{t 0}-x_{t 1}\right\|-\left\|\pi^{* *}\left(x_{t 0}-x_{t 1}\right)\right\| \\
& >\frac{1}{\alpha}-2^{-\left(n_{0}+n+1\right)}>\frac{1}{2 \alpha} .
\end{aligned}
$$

Choose $w_{0}$ and $w_{1}$ in $Y$ such that $\left\|x_{t i}-x_{t}-w_{l}\right\|<2^{-\left(n_{0}+n\right)}$ for $i=0,1$. Let $U$ be the span in $Y^{* *}$ of the 6 vectors $y_{t}, u^{*}\left(x_{t}\right), u^{*}\left(x_{t 0}\right)$, $u^{*}\left(x_{t 1}\right), w_{0}, w_{1}$; let $F$ be the span in $Y^{*}$ of the functionals $f_{1}, \ldots, f_{m}$. By local reflexivity, there is a one-to-one linear operator $T: U \rightarrow Y$ such that $T\left(y_{t}\right)=y_{t}, T\left(w_{i}\right)=w_{l}, i=0,1 ;\left\langle T\left(y^{* *}\right), f_{j}\right\rangle=\left\langle f_{j}, y^{* *}\right\rangle$ for $y^{* *} \in U$ and $1 \leq j \leq m$; and $\|T\|<2,\left\|T^{-1}\right\|<2$. Then

$$
\left\langle f_{j}, T u^{*}\left(x_{t i}-x_{t}\right)\right\rangle=\left\langle f_{j}, u^{*}\left(x_{t i}-x_{t}\right)\right\rangle=\left\langle x_{t i}-x_{t}, f_{j}^{\prime}\right\rangle<1
$$

for $1 \leq j \leq m$, since $x_{t i}-x_{t} \in U_{t}$. Thus $T u^{*}\left(x_{t i}-x_{t}\right) \in V_{t}, i=0,1$. Let $y_{t i}=T u^{*}\left(x_{t i}-x_{t}\right)+y_{t}=T u^{*}\left(x_{t i}-x_{t}+y_{t}\right)$. Then

$$
\begin{aligned}
\left\|y_{t i}-y_{t}-w_{i}\right\| & =\left\|T u^{*}\left(x_{t i}-x_{t}\right)-T\left(w_{i}\right)\right\| \leq 2\left\|u^{*}\left(x_{t i}-x_{t}-w_{i}\right)\right\| \\
& \leq 2\left\|u^{*}\right\| 2^{-\left(n_{0}+n\right)}=\left\|u^{*}\right\| 2^{-\left(n_{0}+n-1\right)} .
\end{aligned}
$$

On the other hand,

$$
\left\|y_{t 0}-y_{t 1}\right\|=\left\|T u^{*}\left(x_{t 1}-x_{t 0}\right)\right\| \geq \frac{1}{2}\left\|u^{*}\left(x_{t 1}-x_{t 0}\right)\right\|>1 / 4 \alpha .
$$

Now $y_{t 0}$ and $y_{t 1}$ belong to $V_{t}+y_{t}$. Choose a basic open neighborhood $V_{t 0}=V_{t 1}$ of 0 in $Y$ so that (1) $w c l\left(y_{t i}+V_{t i}\right) \subseteq y_{t}+V_{t}, i=0,1$; (2) if $z_{i} \in y_{t i}+V_{t i}$, then $\left\|z_{0}-z_{1}\right\|>1 / 4 \alpha$; and (3) $\left(y_{t i}+V_{t i}\right) \cap B$ is contained in an element of $\mathscr{U}_{n+1}$. To complete the construction, we need to verify that $y_{t} \in B$ for each $t$. If $t \in\{0,1\}^{n}$, then

$$
\begin{aligned}
\left\|y_{t}\right\|= & \left\|\sum_{k=1}^{n}\left(y_{t \mid k}-y_{t \mid k-1}\right)\right\| \leq\left\|\sum_{k=1}^{n}\left(x_{t \mid k}-x_{t \mid k-1}\right)\right\| \\
& +\left\|\sum_{k=1}^{n}\left(\left(y_{t \mid k}-y_{t \mid k-1}\right)-\left(x_{t \mid k}-x_{t \mid k-1}\right)\right)\right\| .
\end{aligned}
$$


The first norm is $\left\|x_{t}-x_{\varnothing}\right\| \leq \operatorname{diam}(A)$. A term in the second norm is of the form $\left(y_{s i}-y_{s}\right)-\left(x_{s i}-x_{s}\right)$ where $s \in\{0,1\}^{k}$ and $i=0,1$. But if $w_{i}$ is as above, then

$$
\begin{aligned}
\left\|\left(y_{s i}-y_{s}\right)-\left(x_{s i}-x_{s}\right)\right\| & \leq\left\|y_{s i}-y_{s}-w_{i}\right\|+\left\|x_{s t}-x_{s}-w_{t}\right\| \\
& \leq\left\|u^{*}\right\| 2^{-\left(n_{0}+k-1\right)}+2^{-\left(n_{0}+k\right)} .
\end{aligned}
$$

Thus the norm of the sum is at most

$$
2^{-n_{0}}\left(\sum_{k=1}^{n}\left(\left\|u^{*}\right\| 2^{-(k-1)}+2^{-k}\right)\right) \leq 2^{-n_{0}}\left(2\left\|u^{*}\right\|+1\right)<1,
$$

by the choice of $n_{0}$. Thus $\left\|y_{t}\right\| \leq \operatorname{diam}(A)+1=M$, so $y_{t} \in B$. This completes the recursive construction.

Now let

$$
D=\bigcap_{n=0}^{\infty} \bigcup_{|t|=n}\left(y_{t}+V_{t}\right)
$$

Since wcl $\left(y_{t i}+V_{t l}\right) \subseteq y_{t}+V_{t}$ for all $t$, the set $D$ is weakly closed in $Y$. For each $n, D$ is covered by finitely many sets from $\mathscr{U}_{n}$, and therefore by a single set from $\mathscr{U}_{n}$. If $\mathscr{F}$ is a filter of closed sets in $D$, then $\mathscr{F}$ is $\mathscr{U}_{n}$-small for all $n$, so $\cap \mathscr{F} \neq \varnothing$, by the completeness of the sequence $\left(\mathscr{U}_{n}\right)$. Therefore $D$ is compact. For each $\tau \in\{0,1\}^{\mathbf{N}}$, the set

$$
D_{\tau}=\bigcap_{n=0}^{\infty}\left(y_{\tau \mid n}+V_{\tau \mid n}\right)
$$

is a nonempty subset of $D$. Moreover, if $\tau \neq \sigma, z_{1} \in D_{\tau}, z_{2} \in D_{\sigma}$, then $\left\|z_{1}-z_{2}\right\|>1 / 4 \alpha$. Thus $Y$ is nonseparable, contradicting the fact that $B_{Y}$ is Polish.

5.3. COROLlaRY. If $X$ has $\check{C} e c h$ complete ball, and $X^{* *} / X$ has property (PC), then $X^{* *}$ has property (PC).

Proof. If $X$ has Polish ball, then this follows from 5.2, since $X^{\perp}$ is always complemented in $X^{* * *}$. The general case follows from Theorem B: since $X$ has Čech complete ball, $X=R \oplus S$ where $R$ is reflexive and $S$ has Polish ball. But $S^{* *} / S=X^{* *} / X$ has (PC), so $S^{* *}$ has (PC). The reflexive space $R$ has (PC), so $X=R \oplus S$ has (PC).

As an illustration, consider the dual $\mathrm{JT}^{*}$ of the James Tree space JT. In Example 3.6, we showed that the predual $B$ of JT has Polish ball. But $\mathrm{JT}^{*} / B$ is a nonseparable Hilbert space [38], so it has (PC). Therefore JT* 
has (PC). It is useful to note that $\mathrm{JT}^{*}$ is a nonseparable dual of a separable space, so it fails the RNP [50].

We do not know if hypothesis (3) is necessary for 5.2, nor if (1) can be weakened to " $Y$ has Čech complete ball", or even to " $Y$ has (PC)".

Bourgain [3] has considered a convex version of (PC). We will say that a Banach space $X$ has property $(*)$ iff for each weakly closed bounded convex subset $A$ of $X$, the identity map ( $A$, weak) $\rightarrow(A$, norm) has at least one point of continuity. We do not know if this is equivalent to (PC). An argument similar to 3.11 shows that a Banach space has (*) if and only if every separable subspace has (*). An argument similar to [9] can be given to show that $X$ has $(*)$ if and only if ( $B_{X}$, weak) is huskable for every equivalent norm on $X$. If $X$ is separable, this is equivalent to saying that $B_{X}$ is a Baire space for every equivalent norm on $X$. We omit the proofs.

The paper [55] investigated the conditions under which ( $B_{X}$, weak) satisfies the countable chain condition (every pairwise disjoint collection of nonempty open sets is countable). Theorem 18 of that paper is easily modified to show the following.

5.4. Proposition. Let $X$ be a Banach space with (PC). Then $\left(B_{X}\right.$, weak) satisfies the countable chain condition if and only if $X$ is separable.

Rosenthal [48] proved that for an Eberlein compact, the three conditions (1) separable, (2) metrizable, (3) countable chain condition, are equivalent. Here is a related noncompact theorem.

5.5. Proposition. Let $X$ be a Banach space with $\check{C}$ ech complete ball, $A$ a weakly closed bounded subset. Then the following conditions on ( $A$, weak) are equivalent: (1) separable, (2) metrizable, (3) countable chain condition.

Proof. (1) $\Rightarrow(3)$ is true for all topological spaces. For $(3) \Rightarrow(1)$, modify Theorem 18 of [55].

$(1) \Rightarrow(2)$. Let $Y$ be the closed linear span of $A$. Then $Y$ has Polish ball, so $A$ is metrizable.

$(2) \Rightarrow(1)$. Let $d$ be a metric for ( $A$, weak). If $A$ is not separable, then there is an uncountable set $\left\{a_{i}: i \in I\right\}$ and $\varepsilon>0$ such that $d\left(a_{l}, a_{J}\right)>\varepsilon$ for $i \neq j$. Now $X=R \oplus S$, where $R$ is reflexive and $S$ is separable. Let $a_{l}=r_{i}+s_{i}$ for $i \in I$, where $r_{i} \in R, s_{l} \in S$. Since $S$ is separable, there is a net $\left(s_{i_{\lambda}}\right)$ that converges in norm (perhaps $s_{i_{\lambda}}$ is constant). Since $R$ is reflexive, by taking a subnet we see that $\left(a_{l}\right)$ has a weak cluster point in $A$, a contradiction. 
Here is another application of Čech completeness. A topological space $T$ is said to have a $G_{\delta}$ diagonal iff $\{(t, t): t \in T\}$ is a $G_{\delta}$ set in $T \times T$. A paracompact Čech complete topological space with $G_{\delta}$ diagonal is metrizable [1, p. 37].

5.6. Proposition. Let $A$ be a subset of ( $X$, weak). Then the following are equivalent:

(1) A is Polish;

(2) $A$ is Lindelöf and Čech complete, and there is a countable subset of $X^{*}$ that separates points of $A$.

Proof. (1) $\Rightarrow(2)$. If $Y$ is the closed span of $A$, then $Y^{*}$ is weak* separable.

$(2) \Rightarrow(1)$. The final condition gives $A$ a $G_{\delta}$ diagonal. Thus $A$ is metrizable and Čech complete, so it is Polish.

5.7. ExAmple. Since $\left(l^{\infty}\right)^{*}$ is weak* separable, every Lindelöf Čech complete subset of $\left(l^{\infty}\right.$, weak) is Polish. This is a generalization of the fact that every weakly compact subset of $l^{\infty}$ is separable. Under the Continuum Hypothesis, there is a closed linear subspace $Y$ of $l^{\infty}$ such that every subset is Lindelöf in the weak topology, but $Y$ is not separable [46, Remark 2].

\section{TABLE 1}

\begin{tabular}{|c|c|c|c|c|c|c|c|c|c|}
\hline & & Godefroy & $\begin{array}{c}X^{*} \text { Čech } \\
\text { complete } \\
\text { ball }\end{array}$ & $\begin{array}{c}\text { Čech } \\
\text { complete } \\
\text { ball }\end{array}$ & RNP & PC & $\begin{array}{l}\text { somewhat } \\
\text { reflexive }\end{array}$ & $\begin{array}{l}\text { separably } \\
\text { distinguished }\end{array}$ & Asplund \\
\hline (1) & $B$ & $\mathrm{~N}$ & $\mathrm{~N}$ & Y & $\mathrm{N}$ & Y & Y & Y & Y \\
\hline (2) & $\mathrm{BD}$ & $\mathrm{N}$ & $\mathrm{N}$ & Y & Y & $\mathrm{Y}$ & Y & Y & Y \\
\hline (3) & $c_{0}$ & $\mathrm{~N}$ & $\mathrm{~N}$ & $\mathrm{~N}$ & $\mathrm{~N}$ & $\mathrm{~N}$ & $\mathrm{~N}$ & $\mathrm{Y}$ & Y \\
\hline (4) & $J(\alpha), \alpha<\omega_{1}$ & Y & Y & Y & Y & Y & Y & Y & Y \\
\hline (5) & $J\left(\omega_{1}\right)$ & $\mathrm{N}$ & $\mathrm{N}$ & $\mathrm{N}$ & Y & $\mathrm{Y}$ & Y & $\mathrm{N}$ & Y \\
\hline (6) & $\mathrm{JH}$ & $\mathrm{N}$ & $\mathrm{N}$ & $\mathrm{N}$ & $\mathrm{N}$ & $\mathrm{N}$ & $\mathrm{N}$ & $\mathrm{N}$ & $\mathrm{N}$ \\
\hline (7) & JT & $\mathrm{N}$ & $\mathrm{N}$ & $\mathrm{N}$ & Y & $\mathrm{Y}$ & Y & $\mathrm{N}$ & $\mathrm{N}$ \\
\hline (8) & $\mathrm{JT}^{*}$ & $\mathrm{~N}$ & $\mathrm{~N}$ & $\mathrm{~N}$ & $\mathrm{~N}$ & Y & Y & $\mathrm{N}$ & Y \\
\hline (9) & $l^{1}$ & $\mathrm{~N}$ & $\mathrm{~N}$ & $\mathrm{~N}$ & Y & $\mathrm{Y}$ & $\mathrm{N}$ & $\mathrm{N}$ & $\mathrm{N}$ \\
\hline (10) & $L^{1}[0,1]$ & $\mathrm{N}$ & $\mathrm{N}$ & $\mathrm{N}$ & $\mathrm{N}$ & $\mathrm{N}$ & $\mathrm{N}$ & $\mathrm{N}$ & $\mathrm{N}$ \\
\hline (11) & $\mathrm{JL}\left(c_{0}\right)$ & $Y$ & $Y$ & $Y$ & Y & $\mathrm{Y}$ & Y & $Y$ & Y \\
\hline (12) & $\mathrm{JL}\left(l^{1}\right)$ & $\mathrm{N}$ & $\mathrm{N}$ & Y & Y & $\mathrm{Y}$ & Y & Y & Y \\
\hline (13) & $\mathrm{JL}(\mathrm{JT})$ & Y & $\mathrm{N}$ & Y & $\mathrm{Y}$ & $\mathrm{Y}$ & Y & Y & Y \\
\hline
\end{tabular}

6. Examples. Table 1 displays the properties of several Banach spaces. We include here some brief explanations.

(1) $X=B$, the predual of the James Tree (Example 3.6, [30], [38]). It has property (A), but $B_{X^{* *}} \backslash B_{X}$ is not a countable union of weak* compact convex sets. It has Polish ball but is not isomorphic to a dual 
space, nor complemented in its second dual. It has property (PC), but not RNP. The dual space $B^{*}=$ JT does not have Čech complete ball.

(2) $X=\mathrm{BD}$, a space constructed by Bourgain and Delbaen called $Y$ in [5], [4]. It is a separable $\mathscr{L}^{\infty}$ space, has Polish ball, and has the Dunford-Pettis and Radon-Nikodým properties. The dual $X^{*}$ is isomorphic to $l^{1}$. The space $X$ has no infinite-dimensional reflexive quotients.

(3) $X=c_{0}$ (Example 3.3) is separably distinguished, but does not have Cech complete ball. For the usual norm, $\left(B_{X}\right.$, weak) is first category in itself. For Day's norm, ( $B_{X}$, weak) is a Baire space.

(4) $X=J(\alpha)$, the long James space [17]. If $\alpha$ is a countable ordinal, then $X^{* *}$ is separable, so $X$ has regularly embedded Čech complete ball.

(5) $X=J\left(\omega_{1}\right)$. This nonseparable space has property (PCA), so every separable subspace has Čech complete ball, and $X$ is somewhat reflexive. But $J\left(\omega_{1}\right)$ is not WCG, so its ball is not Čech complete.

(6) $X=\mathrm{JH}$, and James Hagler space [25]. This space $X$ is hereditarily $c_{0}$, and $X^{*}$ is a Schur space. Hence no infinite-dimensional subspace of $X$ or $X^{*}$ has Čech complete ball. The separable subspace $F$ of $\mathrm{JH}^{*}[\mathbf{6}]$ has (PC) but fails the RNP.

(7) $X=\mathrm{JT}$, the James Tree space (Example 3.6, [30], [38]). Now $X$ is separable, $X^{* *} / X$ is reflexive, $X$ is somewhat reflexive, but $B_{X}$ is not Čech complete.

(8) $X=\mathrm{JT}^{*}$, the dual of the James Tree space (Example 3.6, [38], [38]). This space is an Asplund space and has (PC), but not the RNP. Thus every closed bounded subset of ( $\mathrm{JT}^{*}$, weak) is huskable, but this is not true for ( $\mathrm{JT}^{*}$, weak $\left.{ }^{*}\right)$. There is a subspace $B \subseteq X$ with Polish ball such that $X / B$ is reflexive, but $X$ does not have Čech complete ball.

(9) $X=l^{1}$. This space $X$ has the RNP, but $X^{* *}$ fails (PC), since it contains $L^{1}[0,1]$. For any equivalent norm $\left(B_{X}\right.$, weak) is almost Čech complete, but no nonempty $G_{\delta}$ set of $\left(X^{* *}\right.$, weak*) is entirely contained in $X$.

(10) $X=L^{1}[0,1]$. For the usual norm, ( $B_{X}$, weak) is of first category in itself: If $\left(r_{n}\right)$ is the sequence of Rademacher functions, then $B_{X}$ is the union of the closed nowhere dense sets

$$
A_{k}=\left\{f \in B_{X}:\left|\int f(t) r_{n}(t) d t\right| \leq 1 / 4 \text { for all } n \geq k\right\}
$$

For subspaces of $L^{1}$, the properties (PC) and RNP are equivalent. Every subspace of $X$ with separable dual has Polish ball (in fact, reflexive), but $X$ does not have Polish ball. 
(11) $X=\mathrm{JL}\left(c_{0}\right)$, a James-Lindenstrauss space (Example 4.8). If $S$ is separable, the James-Lindenstrauss space $\operatorname{JL}(S)$ can be constructed so as to have property (A). The spaces $\mathrm{JL}(S)$ are separable duals, so they have the RNP. For $X=\mathrm{JL}\left(c_{0}\right)$, the bidual $X^{* *}$ is separable, but $X^{* *} / X$ does not have Čech complete ball. Also, $B_{X^{*}}$ is Čech complete, but $X^{*}$ admits $c_{0}$ as a quotient.

(12) $X=\mathrm{JL}\left(l^{1}\right)$. This space $X$ has Čech complete ball, but $X^{*}$ contains a complemented copy of $l^{1}$, and $X^{* *} / X$ fails the Pettis Integral Property. Also, $X$ is a dual space that has Čech complete ball with a quotient that is not somewhat reflexive. The annihilator $X^{\perp}$ is weak* separable, but $B_{X^{\perp}}$ is not weak* sequentially compact.

(13) $X=\mathrm{JL}(\mathrm{JT})$. Now $B_{X}$ is Čech complete, $X^{*} \nleftarrow l^{1}$, but neither $X^{* *}$ nor $X^{* *} / X$ is measure-compact. Also, $X$ is a Godefroy space, but $B_{X^{*}}$ is not Čech complete.

\section{REFERENCES}

[1] J. Aarts and D. Lutzer, Completeness properties designed for recognizing Baire space, Dissertationes Math., 116 (1974).

[2] D. Amir and J. Lindenstrauss, The structure of weakly compact sets in Banach spaces, Annals of Math., 88 (1968), 35-46.

[3] J. Bourgain, Dentability and finite-dimensional decompositions, Studia Math., 67 (1980), 135-148.

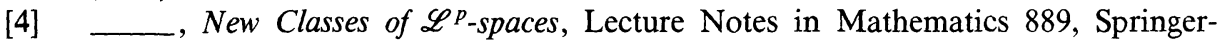
Verlag, 1981.

[5] J. Bourgain and F. Delbaen, $A$ class of special $\mathscr{L}^{\infty}$-spaces, Acta Math., 145 (1980), 155-176.

[6] J. Bourgain and H. Rosenthal, Geometrical implications of certain finite dimensional decompositions, Bull. Soc. Math. Belg., 32 (1980), 57-82.

[7] J. Clark, Coreflexive and somewhat reflexive Banach spaces, Proc. Amer. Math. Soc., 36 (1972), 421-427.

[8] H. Corson, The weak topology of a Banach space, Trans. Amer. Math. Soc., 101 (1961), 1-15.

[9] W. Davis and R. Phelps, The Radon-Nikodym property and dentable sets in Banach spaces, Proc. Amer. Math. Soc., 45 (1974), 119-122.

[10] W. Davis and I. Singer, Boundedly complete M-bases and complemented subspaces in Banach spaces, Trans. Amer. Math. Soc., 175 (1973), 187-194.

[11] J. Diestel, Geometry of Banach Spaces-Selected Topics, Lecture Notes in Mathematics 485, Springer-Verlag, 1975.

[12] A survey of results related to the Dunford-Pettis property, Proc. Conf. on Integration, Topology, and Geometry in Linear Spaces, Amer. Math. Soc. Contemp. Mathematics 2, (1980), 15-60.

[13] J. Diestel and J. Uhl, Vectors Measures, Amer. Math. Soc. Math. Surveys 15, American Mathematical Society, 1977.

[14] N. Dunford and J. Schwartz, Linear Operators, Part I, Interscience, 1957. 
[15] G. Edgar, Measurability in a Banach space, Indiana Univ. Math. J., 26 (1977), 663-677.

[16] _ Measurability in a Banach space II, Indiana Univ. Math. J., 28 (1979), $559-579$.

[17] _ A Long James Space, Measure Theory, Oberwolfach 1979, D. Kölzow (editor), Lecture Notes in Mathematics 794, Springer-Verlag, (1980), 31-37.

[18] R. Engelking, General Topology, PWN-Polish Scientific Publishers, Warsaw, 1977.

[19] Z. Frolik, Generalizations of the $G_{\delta}$ property of complete metric spaces, Czech. Math. J., 10 (85) (1960), 359-378.

[20] _ Baire spaces and some generalizations of complete metric spaces, Czech. Math. J., 11 (86) (1961), 237-247.

[21] G. Godefroy, Epluchabilité et unicité du prédual, Sem. Choquet 1977/78, Comm. \#11.

[22] Espaces de Banach: existence et unicité de certains préduaux, Ann. Inst. Fourier (Grenoble), 28, no. 3 (1978), 87-105.

[23] _ Compacts de Rosenthal, Pacific J. Math., 91 (1980), 293-306.

[24] W. Govaerts, A productive class of angelic spaces, J. London Math. Soc., (2) 22 (1980), 355-364.

[25] J. Hagler, A counterexample to several questions about Banach spaces, Studia Math., 60 (1977), 289-308.

[26] J. Hagler and W. Johnson, On Banach spaces whose dual balls are not weak* sequentially compact, Israel J. Math., 28 (1977), 325-330.

[27] R. Haworth and R. McCoy, Baire Spaces, Dissertationes Math., 141 (1977).

[28] W. Hurewicz, Relative perfekte Teile von Punktmengen und Mengen (A), Fund. Math., 12 (1928), 78-109.

[29] R. James, Separable conjugate spaces, Pacific J. Math., 10 (1960), 563-571.

[30] _ A separable somewhat reflexive Banach space with nonseparable dual, Bull. Amer. Math. Soc., 80 (1974), 738-743.

[31] W. Johnson and H. Rosenthal, On $\omega^{*}$-basic sequences and their applications to the study of Banach spaces, Studia Math., 43 (1972), 77-92.

[32] W. Johnson, H. Rosenthal, and M. Zippin, On bases, finite-dimensional decompositions, and weaker structures in Banach spaces, Israel J. Math., 9 (1971), 488-506.

[33] P. Kenderov, Dense strong continuity of pointwise continuous mappings, Pacific J. Math., 89 (1980), 111-130.

[34] J. Kerstan, Eine Klasse topologischer Räume, die die polnischen Räume und die o-kompakten lokalkompakten Hausdorffschen Räume verallgemeinern, Theory of Sets and Topology, G. Asser, J. Flachsmeyer, and W. Rinow (editors), VEB Deutscher Verlag der Wissenschaften, 1972, pp. 291-311.

[35] T. Kuo, On conjugate Banach spaces with the Radon-Nikodym property, Pacific J. Math., 59 (1975), 497-503.

[36] J. Lindenstrauss, On James' paper “Separable conjugate spaces”, Israel J. Math., 9 (1971), 279-284.

[37] Weakly compact sets - their topological properties and the Banach spaces they generate, Proc. Sympos. Infinite-Dimensional Topology, Ann. of Math. Studies 69, Princeton University Press, (1972), 235-293.

[38] J. Lindenstrauss and C. Stegall, Examples of separable spaces which do not contain $l^{1}$ and whose duals are non-separable, Studia Math., 54 (1975), 81-105.

[39] J. Lindenstrauss and L. Tzafriri, Classical Banach Spaces, I. Sequence Spaces, Ergebnisse der Mathematik und Ihrer Grenzgebiete 92, Springer-Verlag, 1977.

[40] H. Maynard, A geometrical characterization of Banach spaces with the Radon-Nikodym property, Trans. Amer. Math. Soc., 185 (1973), 493-500. 
[41] R. McWilliams, On certain Banach spaces which are weak*-sequentially dense in their second duals, Duke Math. J., 37 (1970), 121-126.

[42] I. Namioka, Separate continuity and joint continuity, Pacific J. Math., 51 (1974), 515-531.

[43] E. Odell and H. Rosenthal, $A$ double-dual characterization of separable Banach spaces containing $l^{1}$, Israel J. Math., 20 (1975), 375-384.

[44] R. Pol, Concerning function spaces on separable compact spaces, Bull. Acad. Polon. Sci., 25 (1977), 993-997.

[45] On a question of $H$. H. Corson and some related problems, Fund. Math., 109 (1980), 143-154.

[46] The Lindelöf property and its convex analogue in function spaces with the weak topology, Topology, vol. II, A. Csaszar (editor), North-Holland, (1980), 965-969.

[47] D. Preiss, Metric spaces in which Prohorov's theorem is not valid, Z. Wahrscheinlichkeitstheorie und Verw. Gebiete, 27 (1973), 109-116.

[48] H. Rosenthal, On injective Banach spaces and the spaces $L^{\infty}(\mu)$ for finite measures $\mu$, Acta Math., 124 (1970), 205-248.

[49] J. Saint-Raymond, La structure borélienne d'Effros est-elle standard?, Fund. Math., 100 (1978), 201-210.

[50] C. Stegall, The Radon-Nikodym property in conjugate Banach spaces I, Trans. Amer. Math. Soc., 206 (1975), 213-223.

[51] The Radon-Nikodym property in conjugate Banach spaces II, Trans. Amer. Math. Soc., 264 (1981), 507-519.

[52] M. Talagrand, Sur une conjecture de H. H. Corson, Bull. Sci. Math., (2) 99 (1975), 211-212.

[53] F. Topsøe and J. Hoffmann-Jørgensen, Analytic Spaces and Their Application, Analytic Sets, C. A. Rogers, et. al., Academic Press, 1980.

[54] M. Valdivia, On a class of Banach spaces, Studia Math., 60 (1977), 11-13.

[55] R. Wheeler, The retraction property, CCC property, and Dunford-Pettis-Phillips property for Banach spaces, Measure Theory, Oberwolfach 1981, D. Kölzow (editor), Lecture Notes in Mathematics 945, Springer-Verlag, 1982, pp. 252-262.

[56] A. Wilansky, Modern Methods in Topological Vector Spaces, McGraw-Hill, 1978.

Received March 23, 1983.

The OHIO STATE UNIVERSITY

Columbus, OH 43210

AND

NORTHERN ILLINOIS UNIVERSITY

DeKalb, IL 60115 


\title{
PACIFIC JOURNAL OF MATHEMATICS EDITORS
}

\author{
Donald BABBITt (Managing Editor) \\ University of California \\ Los Angeles, CA 90024 \\ J. DugunduI \\ University of Southern California \\ Los Angeles, CA 90089-1113 \\ R. FINN \\ Stanford University \\ Stanford, CA 94305 \\ HermanN FlaschKa \\ University of Arizona \\ Tucson, AŻ 85721
}

C. C. Moore

University of California

Berkeley, CA 94720

ARTHUR Ogus

University of California

Berkeley, CA 94720

Hugo Rossi

University of Utah

Salt Lake City, UT 84112

H. SAMELSON

Stanford University

Stanford, CA 94305

ASSOCIATE EDITORS
R. ARENS
E. F. BECKENBACH
B. H. NEUMANN
F. WOLF
K. YoSHIDA (1906-1982)

\section{SUPPORTING INSTITUTIONS}

$\begin{array}{ll}\text { UNIVERSITY OF ARIZONA } & \text { UNIVERSITY OF OREGON } \\ \text { UNIVERSITY OF BRITISH COLUMBIA } & \text { UNIVERSITY OF SOUTHERN CALIFORNIA } \\ \text { CALIFORNIA INSTITUTE OF TECHNOLOGY } & \text { STANFORD UNIVERSITY } \\ \text { UNIVERSITY OF CALIFORNIA } & \text { UNIVERSITY OF HAWAII } \\ \text { MONTANA STATE UNIVERSITY } & \text { UNIVERSITY OF TOKYO } \\ \text { UNIVERSITY OF NEVADA, RENO } & \text { UNIVERSITY OF UTAH } \\ \text { NEW MEXICO STATE UNIVERSITY } & \text { WASHINGTON STATE UNIVERSITY } \\ \text { OREGON STATE UNIVERSITY } & \text { UNIVERSITY OF WASHINGTON }\end{array}$

The Supporting Institutions listed above contribute to the cost of publication of this Journal, but they are not owners or publishers and have no responsibility for its content or policies.

Mathematical papers intended for publication in the Pacific Journal of Mathematics should be in typed form or offset-reproduced (not dittoed), double spaced with large margins. Please do not use built up fractions in the text of the manuscript. However, you may use them in the displayed equations. Underline Greek letters in red, German in green, and script in blue. The first paragraph must be capable of being used separately as a synopsis of the entire paper. In particular it should contain no bibliographic references. Please propose a heading for the odd numbered pages of less than 35 characters. Manuscripts, in triplicate, may be sent to any one of the editors. Please classify according to the scheme of Math. Reviews, Index to Vol. 39. Supply name and address of author to whom proofs should be sent. All other communications should be addressed to the managing editor, or Elaine Barth, University of California, Los Angeles, California 90024.

There are page-charges associated with articles appearing in the Pacific Journal of Mathematics. These charges are expected to be paid by the author's University, Government Agency or Company. If the author or authors do not have access to such Institutional support these charges are waived. Single authors will receive 50 free reprints; joint authors will receive a total of 100 free reprints. Additional copies may be obtained at cost in multiples of 50 .

The Pacific Journal of Mathematics is issued monthly as of January 1966. Regular subscription rate: $\$ 190.00$ a year (5 Vols., 10 issues). Special rate: $\$ 66.00$ a year to individual members of supporting institutions.

Subscriptions, orders for numbers issued in the last three calendar years, and changes of address should be sent to Pacific Journal of Mathematics, P.O. Box 969, Carmel Valley, CA 93924, U.S.A. Old back numbers obtainable from Kraus Periodicals Co., Route 100, Millwood, NY 10546.

The Pacific Journal of Mathematics at P.O. Box 969, Carmel Valley, CA 93924 (ISSN 0030-8730) publishes 5 volumes per year. Application to mail at Second-class postage rates is pending at Carmel Valley, California, and additional mailing offices. Postmaster: Send address changes to Pacific Journal of Mathematics, P.O. Box 969, Carmel Valley, CA 93924.

PUBLISHED BY PACIFIC JOURNAL OF MATHEMATICS, A NON-PROFIT CORPORATION

Copyright $@ 1984$ by Pacific Journal of Mathematics 


\section{Pacific Journal of Mathematics}

\section{Vol. 115, No. $2 \quad$ October, 1984}

Ersan Akyildiz, Gysin homomorphism and Schubert calculus ...........257

Marilyn Breen, Clear visibility and unions of two starshaped sets in the

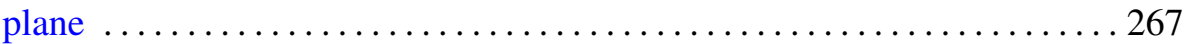

Robert F. Brown, Retraction methods in Nielsen fixed point theory . ......277

Herbert Busemann and Bhalchandra B. Phadke, A general version of Beltrami's theorem in the large ............................... 299

Gerald Arthur Edgar and Robert Francis Wheeler, Topological properties of Banach spaces ............................... 317

Yaakov Friedman and Bernard Russo, Conditional expectation without

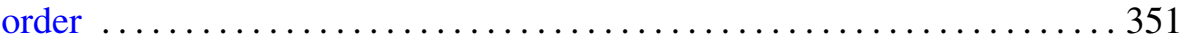

Robert Allen Goggins, Cobordism of manifolds with strong almost tangent

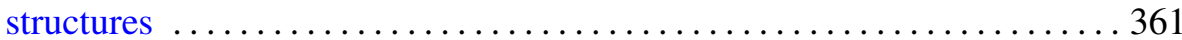

Mike Hoffman, Noncoincidence index of manifolds . . . . . . . . . . . . . 373

William H. Julian, $\varepsilon$-continuity and monotone operations $\ldots \ldots \ldots \ldots 385$

Gerasimos E. Ladas, Y. G. Sficas and I. P. Stavroulakis, Nonoscillatory

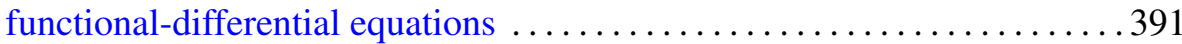

Arnold William Miller and Karel Libor Prikry, When the continuum has

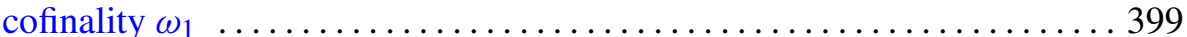

Jean-Leah Mohrherr, Density of a final segment of the truth-table

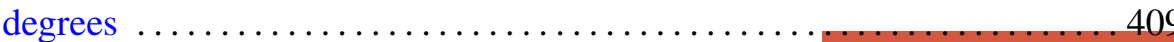

Carl Norman Mutchler, The flat Cauchy problem for radially hyperbolic operators from a characteristic manifold of high codimension ...

Kenji Nakagawa, On the orders of automorphisms of a closed Riemann surface

W. Ricker, Representation of vector-valued functions by Laplace transforms

Jorge Donato Samur, On semigroups of convolution operators in Hilbert

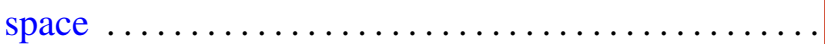

Joseph Gail Stampfli, One-dimensional perturbations of operators 481 Andrew George Earnest and John Sollion Hsia, Correction to: "Spinor norms of local integral rotations. II" 